\title{
A Regulator for Continuous Sedimentation in Ideal Clarifier-Thickener Units
}

\author{
STEFAN DIEHL \\ Centre for Mathematical Sciences, Lund University, P.O. Box 118, SE-221 00 Lund, Sweden (e-mail: \\ diehl@maths.lth.se)
}

\begin{abstract}
The purpose of this paper is to present a regulator for control of the continuous-sedimentation process in a clarifier-thickener unit when this is modelled in one space dimension and when the settling properties of the solids obey Kynch's assumption. The model is a scalar hyperbolic conservation law with space-discontinuous flux function and point source. The most desired type of solution contains a large discontinuity. A common objective is to control the movement of this discontinuity subject to the requirement that the effluent of the process have zero concentration of particles. In addition, there may be a requirement that the underflow concentration of the thickened suspension lie above a predefined value. Based on previous results on the nonlinear behaviour of the process, a nonlinear regulator is presented. It controls the location of the large discontinuity indirectly by controlling the total mass. The process is stabilized significantly and large input oscillations can be handled.
\end{abstract}

Keywords: continuous sedimentation, control, nonlinear regulator, dynamic behaviour, clarifier-thickener

\section{Introduction}

The aim of the process of continuous sedimentation is to separate particles from a liquid in a large tank under a continuous inflow of mixture at an intermediate feed level. The particles settle by gravity and are also influenced by a bulk flow upwards above the feed inlet (the clarification zone), and a bulk flow downwards below the feed inlet (the thickening zone), see Figure 1 (left). Under optimal operating conditions, there is a discharge of a highly concentrated suspension at the bottom (the underflow) simultaneously with a clarified overflow of liquid at the top of the tank (the effluent). The continuous-sedimentation tank is widely used in mineral processing, wastewater treatment plants, chemical engineering etc., and is called clarifier-thickener unit, or settler. Under optimal operating conditions there are no particles in the clarification zone and a large discontinuity in the thickening zone, called the sludge blanket in wastewater treatment. This state of the settler is called optimal operation.

The process has been used for about a century and is well known to be nonlinear, which is why its behaviour is difficult to predict as well as to model. The need to control the process for obtaining a clarified effluent is obvious. In a wastewater treatment plant most of the concentrated underflow, which is biological sludge, is recycled within the plant to a biological reactor that precedes the settler. Therefore, it is also vital to be able to control the underflow concentration. In such an activated sludge system the settler also serves as a buffer of biological sludge. These aspects can be fulfilled by controlling the sludge blanket level.

Independently of the application, the process is highly nonlinear even under the most common idealized assumptions, which are the following. The clarifier-thickener unit is ideal in the sense that all flows in the tank occur only in one dimension, the feed inlet is a point source, the cross-sectional area is constant and the concentration is constant on each cross-section. Furthermore, the particles are assumed to be equally-sized spheres that form a non-compressible sediment at a maximum concentration. 
In the series of papers [1-4] thorough investigations have provided a deeper knowledge as well as classifications of the nonlinear behaviour of an idealized one-dimensional clarifierthickener model. The hyperbolic PDE-model was formulated and analyzed in [5, 6], in which existence and uniqueness locally in time were proved. Global existence and uniqueness were established by Bürger et al. [7, 8] and Karlsen and Towers [9]. The PDE-model is hyperbolic because of the constitutive assumption by Kynch [10]: the settling flux of particles is a function only of the concentration. We refer to the series [1-4] for justifications, discussions and references regarding the present model as well as the parallel 'engineering' development without PDEs. We mention only the recent important contributions by Bürger et al. [11, 12], which rely on the analyses by Karlsen et al. $[13,14]$. They formulate and analyze a more general PDE model which includes compression at high concentrations.

Analyses, based on PDE solutions, of the possibilities of controlling the sludge blanket (large discontinuity) can be found in [15-21]. In all these references it is assumed that the variation of the feed input is so moderate that a quasi steady-state situation remains with a sludge blanket. The limitations for control are analyzed by the author in [4]. An interesting simplified, lumped parameter model was presented in a short but comprehensive paper by Stehfest [15] in 1984. In contrast to several other published models (not referred to here), the arguments behind Stehfest's model, in particular considering the boundary conditions, agree with the theory of conservation laws with space-discontinuous flux functions that was developed during the 1990's ([22, 5, 6, 23]). The step responses presented in [15] agree with those in Section 3.2 in the present paper. Chancelier et al. [19,20] use a feedback law to control the sludge blanket. However, it requires that the sludge blanket level is measured, which may be difficult. In the present paper we show how the sludge blanket can be controlled without measuring its location.

The need to control the settler is also emphasized, directly or indirectly, in the applications, see e.g. [24-46].

For step inputs, optimal control strategies were presented in [3] in order to meet the different control objectives suggested. Those control objectives are exhaustive in the sense that they can always be met, also for theoretically possible but maybe unrealistically high values of the feed variables. In [4], it was shown there how the process could be controlled, however, not automatically. There is a need for a refined control strategy for fine-tuning the sludge blanket level. This is taken care of in the present paper.

In Section 2, the process, the model and the previous results are reviewed briefly. The main condition of the previously presented control objectives - to maintain optimal operation as long as possible - is now refined to include control of the sludge blanket, possibly with a constraint on the underflow concentration, see Section 3.1. Section 3 contains justifications and reasons behind the control strategy, which can be found in Section 3.4. The strategy means that the sludge blanket level is controlled indirectly by controlling the mass in the settler. The strategy is realized by a proportional regulator in Section 4, which works for moderately varying indata. For indata with large oscillations, the proportional regulator has to saturate according to the limitations of the control variable presented in [4]. The complete nonlinear regulator can be found in Section 5, which concludes with several simulations illustrating some properties of the regulator. 


\section{Preliminaries}

We review only briefly the fundamental notation and results presented in the papers [1-4]. These concepts are sufficient for understanding the ideas, results and simulations of the paper. For the details of construction of the solutions shown in Section 3.2, we refer to [2, Section 2]. For the numerical simulations we use the data and batch-settling flux function given in the caption of Figure 1 and the numerical method in [47].

\subsection{THE CLARIFIER-THICKENER UNIT AND THE MODEL}

The one-dimensional model of the clarifier-thickener unit, or settler, was first presented in [6]. Figure 1 shows the settler and the flux function in the thickening zone for three different values of the control parameter $Q_{\mathrm{u}}$. The purposes of the settler may vary depending on in
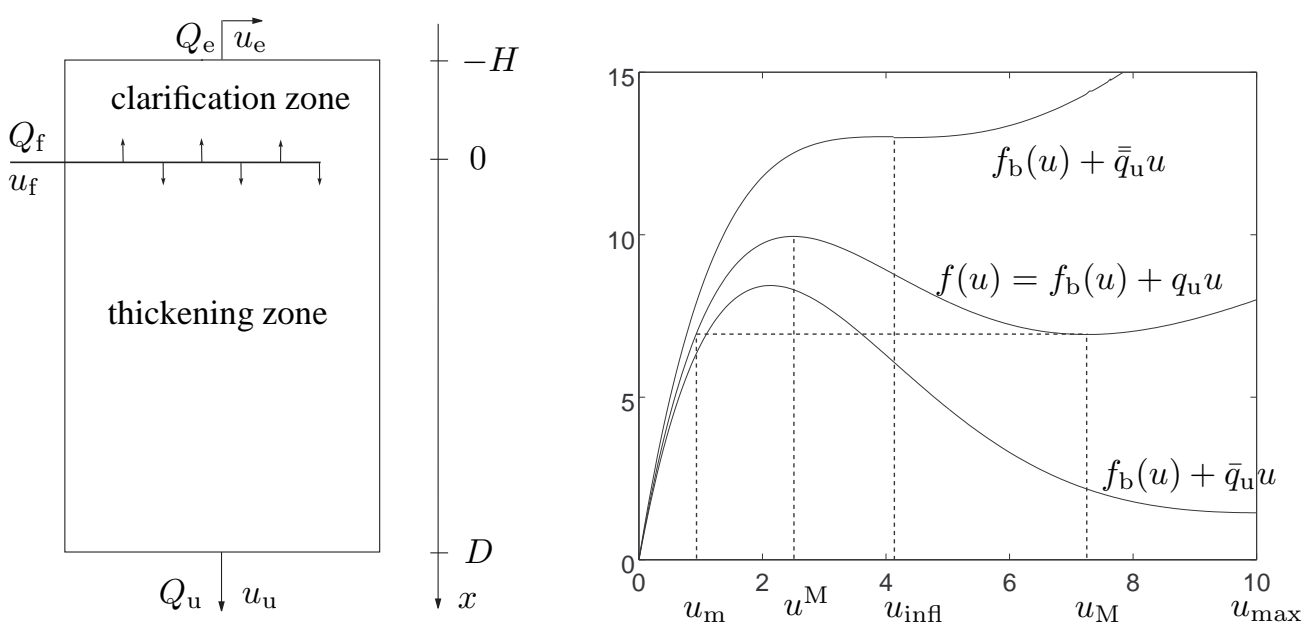

Figure 1. Left: Schematic picture of an ideal one-dimensional clarifier-thickener unit, where $u$ stands for concentration and $Q$ for volume flow of the feed, effluent and underflow streams, respectively. The flow restrictions are $Q_{\mathrm{f}}=Q_{\mathrm{e}}+Q_{\mathrm{u}}>0$ and $Q_{\mathrm{e}} \geq 0$. For the numerical simulations we use $H=1 \mathrm{~m}, D=4 \mathrm{~m}$, and $A=\pi 30^{2} \mathrm{~m}^{2} \approx 2827 \mathrm{~m}^{2}$ for the constant cross-sectional area. Right: Flux curves $f(u)$ in the thickening zone and characteristic concentrations. The bulk velocities are defined as $q_{\mathrm{e}}=Q_{\mathrm{e}} / A$ etc. The constant $u_{\text {inff }}$ is the inflection point of $f_{\mathrm{b}}(u)$ and $f(u)=f_{\mathrm{b}}(u)+q_{\mathrm{u}} u$. For $\bar{q}_{\mathrm{u}}<q_{\mathrm{u}}<\overline{\bar{q}}_{\mathrm{u}}$ there is a local minimum point $u_{\mathrm{M}}$ of $f(u)$ that lies between $u_{\text {inf }}$ and $u_{\text {max }}$. Given $u_{\mathrm{M}}, u_{\mathrm{m}}$ is the lower concentration defined by $f\left(u_{\mathrm{m}}\right)=f\left(u_{\mathrm{M}}\right)$. For $q_{\mathrm{u}}<\overline{\bar{q}}_{\mathrm{u}}$ there is a local maximum point, $u^{\mathrm{M}}\left(<u_{\mathrm{infl}}\right)$ of $f(u)$. The batch-settling flux used for demonstrations with numerical simulations is $f_{\mathrm{b}}(u)=10 u\left(\left(1-0.64 u / u_{\max }\right)^{6.55}-0.36^{6.55}\right)\left[\mathrm{kg} /\left(\mathrm{m}^{2} \mathrm{~h}\right)\right]$.

what industrial process it is involved. At least in wastewater treatment the main purposes of the settler are the following. It should

1. produce a low effluent concentration;

2. produce a high underflow concentration;

3. work as a buffer of mass and be insensitive to small variations in the feed variables.

The one-dimensional model of the settler is the following. The conservation law can be written as the partial differential equation

$$
u_{t}+(F(u, x, t))_{x}=s(t) \delta(x),
$$


where $\delta$ is the Dirac measure, the total flux function is

$$
F(u, x, t)= \begin{cases}-q_{\mathrm{e}}(t) u, & x<-H \\ g\left(u, Q_{\mathrm{e}}(t)\right)=f_{\mathrm{b}}(u)-q_{\mathrm{e}}(t) u, & -H<x<0 \\ f\left(u, Q_{\mathrm{u}}(t)\right)=f_{\mathrm{b}}(u)+q_{\mathrm{u}}(t) u, & 0<x<D \\ q_{\mathrm{u}}(t) u, & x>D,\end{cases}
$$

and the source function is

$$
s(t)=\frac{Q_{\mathrm{f}}(t)}{A} u_{\mathrm{f}}(t)=\frac{Q_{\mathrm{u}}(t)+Q_{\mathrm{e}}(t)}{A} u_{\mathrm{f}}(t)=\left(q_{\mathrm{u}}(t)+q_{\mathrm{e}}(t)\right) u_{\mathrm{f}}(t) .
$$

For convenience, the dependences of the flux functions within the settler on the (time varying) volume flows are only written out when it is needed, i.e., $f(u)=f\left(u, Q_{\mathrm{u}}(t)\right)$. The physical input variables are the feed concentration $u_{\mathrm{f}}$ and the feed volume flow $Q_{\mathrm{f}}$. For graphical interpretations in operating charts it is, however, convenient to use the feed point $\left(u_{\mathrm{f}}, s\right)$ as input variable. The control variable of the process is $Q_{\mathrm{u}}$ and has the natural restriction $0<Q_{\mathrm{u}} \leq Q_{\mathrm{f}}$. Two particular values of this variable arise from the properties of the batch settling flux function. Define

$$
\begin{array}{ll}
\bar{q}_{\mathrm{u}}=-f_{\mathrm{b}}^{\prime}\left(u_{\max }\right), & \bar{Q}_{\mathrm{u}}=\bar{q}_{\mathrm{u}} A, \\
\overline{\bar{q}}_{\mathrm{u}}=-f_{\mathrm{b}}^{\prime}\left(u_{\mathrm{infl}}\right), & \overline{\bar{Q}}_{\mathrm{u}}=\overline{\bar{q}}_{\mathrm{u}} A,
\end{array}
$$

which are the bulk velocities such that the slope of $f$ is zero at $u_{\max }$ and $u_{\text {infl }}$, respectively, see Figure 1 (right).

\subsection{OPERATING CHARTS AND OPTIMAL OPERATION}

Figure 2 shows the 'steady-state chart' and the 'control chart'. Depending on the location of the
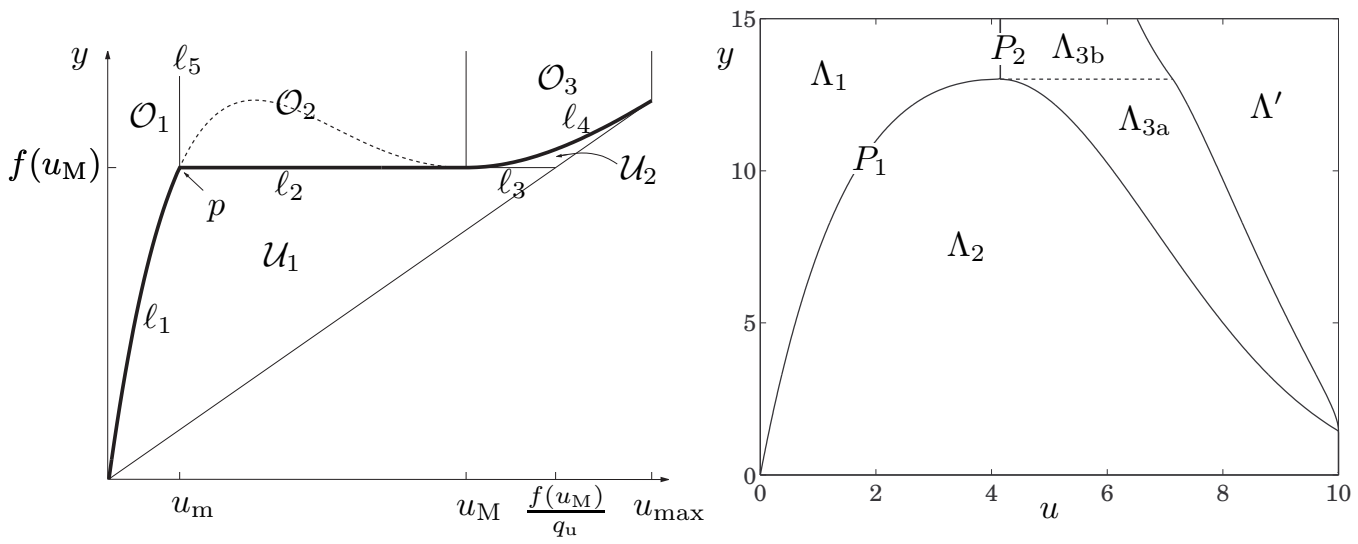

Figure 2. Left: The steady-state chart. The thick graph is the limiting flux curve. If the feed point lies on this curve, the settler is critically loaded in steady state, which means that it works at its maximum capacity. Below this graph the settler is underloaded, and above it is overloaded with a non-zero effluent concentration. Each region corresponds to a specific steady state which is unique, except on the limiting flux curve (and on $\ell_{3}$ and $\ell_{5}$ ), where the location of a discontinuity in the thickening and/or the clarification zone is not uniquely determined. Note that the regions in this chart all depend on $Q_{\mathrm{u}}$. Right: The control chart with respect to steady states; $\Lambda_{3}=\Lambda_{3 \mathrm{a}} \cup \Lambda_{3 \mathrm{~b}}$, $\Lambda_{4}=\Lambda_{3} \cup \Lambda^{\prime}$. The regions in this chart are fixed (given the batch settling flux $f_{\mathrm{b}}$ ). 
feed point $\left(u_{\mathrm{f}}, s\right)$ in the steady-state chart, there are different possible steady-state solutions, which are all piecewise constant and non-decreasing with depth; see [1, Table 1] for a complete table. The limiting flux is defined as:

$$
f_{\lim }(u)=\min _{u \leq \alpha \leq u_{\max }} f(\alpha)= \begin{cases}f(u), & u \in\left[0, u_{\mathrm{m}}\right] \cup\left[u_{\mathrm{M}}, u_{\max }\right] \\ f\left(u_{\mathrm{M}}\right), & u \in\left(u_{\mathrm{m}}, u_{\mathrm{M}}\right)\end{cases}
$$

see Figure 2 (left). This flux, as well as the characteristic concentrations and the regions of the steady-state chart, depend on the control variable $Q_{\mathrm{u}}$; e.g. $u_{\mathrm{M}}\left(Q_{\mathrm{u}}\right), f\left(u, Q_{\mathrm{u}}\right)$ and $f_{\lim }\left(u_{\mathrm{f}}, Q_{\mathrm{u}}\right)$. The following regions in the operating chart are independent of $Q_{\mathrm{u}}$ :

$$
\begin{aligned}
\Lambda_{i} & =\bigcup_{Q_{\mathrm{u}}>0} \ell_{i}\left(Q_{\mathrm{u}}\right), \quad i=1, \ldots, 4, \\
P & =P_{1} \cup P_{2}, \quad \text { where } P_{1}=\bigcup_{0<Q_{\mathrm{u}} \leq \overline{\bar{Q}}_{\mathrm{u}}} p\left(Q_{\mathrm{u}}\right), \quad P_{2}=\bigcup_{Q_{\mathrm{u}}>\overline{\bar{Q}}_{\mathrm{u}}} p\left(Q_{\mathrm{u}}\right), \\
\Lambda_{3 \mathrm{a}} & =\Lambda_{3} \cap\left\{(u, y): y<f_{\mathrm{b}}\left(u_{\text {infl }}\right)+\overline{\bar{q}}_{\mathrm{u}} u_{\text {infl }}\right\} \quad \text { and } \quad \Lambda_{3 \mathrm{~b}}=\Lambda_{3} \backslash \Lambda_{3 \mathrm{a}},
\end{aligned}
$$

see Figure 2 (right). Given a feed point in this chart, there is a unique graph $f_{\lim }\left(\cdot, \tilde{Q}_{\mathrm{u}}\right)$ that passes through the feed point, see [1, Theorem 2]. With this unique value $\tilde{Q}_{\mathrm{u}}$ on the control parameter, the settler is critically loaded in steady state, which means that any higher load (mass per time unit) fed to the settler will result in an overflow of particles.

A more important concept than critically loaded is optimal operation. These concepts are related but not identical. Optimal operation in steady state means that the concentration is zero in the clarification zone and there is a discontinuity in the thickening zone between the concentrations $u_{\mathrm{m}}$ and $u_{\mathrm{M}}$, see Figure 1 (right). This discontinuity is, in wastewater treatment, called the sludge blanket and its location at the depth $x=x_{\mathrm{sb}} \in(0, D)$ is called the sludge blanket level (SBL). A rising SBL refers to reality, although the $x$-coordinate decreases, because of the downward-pointing $x$-axis. A necessary condition for this state is that $\left(u_{\mathrm{f}}, s\right) \in p\left(Q_{\mathrm{u}}\right) \cup \ell_{2}\left(Q_{\mathrm{u}}\right) \cup \ell_{3}\left(Q_{\mathrm{u}}\right)$ and $Q_{\mathrm{u}}<\overline{\bar{Q}}_{\mathrm{u}}$, which implies $\left(u_{\mathrm{f}}, s\right) \in P_{1} \cup \Lambda_{2} \cup \Lambda_{3 \mathrm{a}}$, see Figure 2.

For a general dynamic solution, optimal operation and the SBL are defined as follows. Let $u_{\mathrm{cl}}$ denote the restriction of the solution $u$ to the clarification zone.

DEFINITION 2.1. The settler is said to be in optimal operation at time $t$ if $Q_{\mathrm{u}}(t)<\overline{\bar{Q}}_{\mathrm{u}}$ and the solution of (1) satisfies:

- $u_{\mathrm{cl}}(x, t)=0 \Leftrightarrow u(x, t)=0,-H<x<0$,

- there exists a level $x_{\mathrm{sb}}(t) \in(0, D)$ such that

$$
u(x, t) \in \begin{cases}{\left[0, u_{\text {infl }}\right),} & 0<x<x_{\mathrm{sb}}(t) \\ {\left[u_{\text {infl }}, u_{\max }\right],} & x_{\mathrm{sb}}(t)<x<D .\end{cases}
$$

The definition implies a natural definition of the SBL for a settler in optimal operation: it is the discontinuity at the depth $x=x_{\mathrm{sb}}(t)$ in the thickening zone, such that the jump in the concentration passes the characteristic concentration $u_{\text {inff }}$. It is convenient to use this definition of the SBL also when there are particles in the clarification zone. 
In the analyses of step responses and control of these, it turned out that similar lines to the graph of the limiting flux were convenient to introduce. With the same notation as in the previous papers, we define (and skip $L_{2}$ since we do not need it here):

$$
\begin{aligned}
& L_{1}=\bigcup_{i=1}^{3} \ell_{i} \cup p \cup\left\{(u, y): y=q_{\mathrm{u}} u, \frac{f\left(u_{\mathrm{M}}\right)}{q_{\mathrm{u}}}<u \leq u_{\max }\right\} \\
& L_{3}=\left\{(u, y): y=f_{3}(u)\right\} \quad \text { where } f_{3}(u)= \begin{cases}f(u), & 0 \leq u \leq u^{\mathrm{M}} \\
f\left(u^{\mathrm{M}}\right), & u^{\mathrm{M}}<u \leq \frac{f\left(u^{\mathrm{M}}\right)}{q_{\mathrm{u}}} \\
q_{\mathrm{u}} u, & \frac{f\left(u^{\mathrm{M}}\right)}{q_{\mathrm{u}}}<u \leq u_{\max }\end{cases}
\end{aligned}
$$

Note that these sets depend on $Q_{\mathrm{u}}$, see Figure 3. By the control strategy DCL1 (direct control
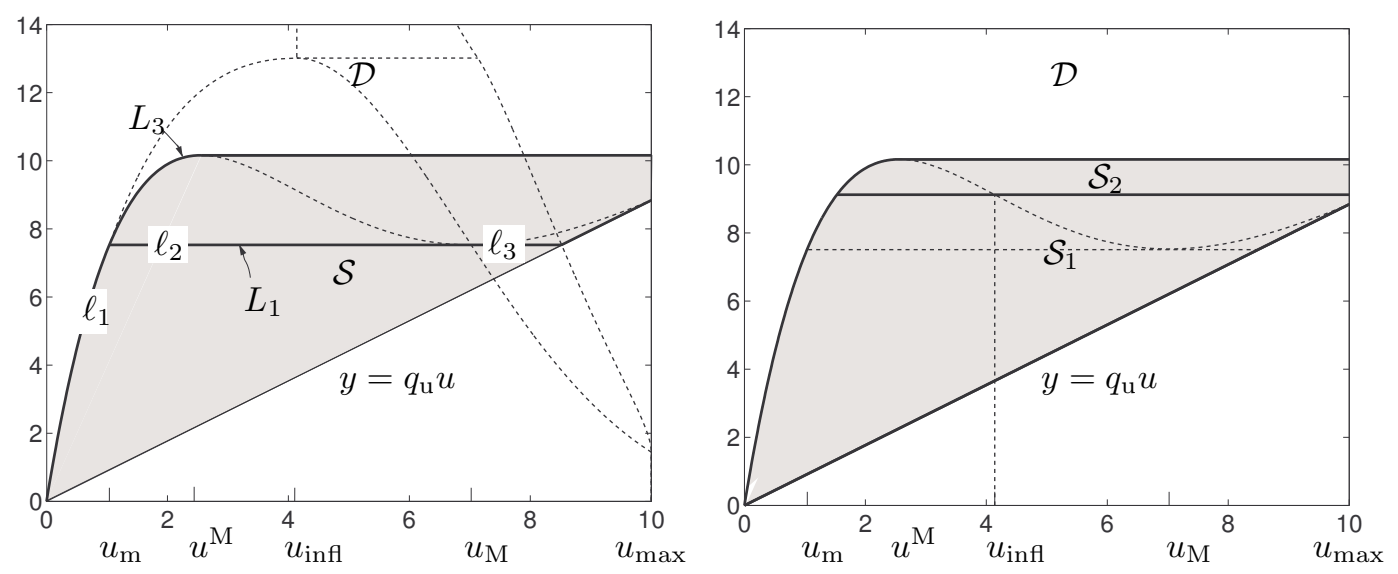

Figure 3. The set $\mathcal{S}=\mathcal{S}_{1} \cup \mathcal{S}_{2}$ (the 'safe' region) is the closed region below $L_{3}$, shaded in the figure. $\mathcal{D}$ (the 'dangerous' region) is its complement, i.e. it lies strictly above $L_{3}$. (Note that the feed point has to lie on or above the line $y=q_{\mathrm{u}} u$, since $s=Q_{\mathrm{f}} u_{\mathrm{f}} / A \geq Q_{\mathrm{u}} u_{\mathrm{f}} / A=q_{\mathrm{u}} u_{\mathrm{f}}$.)

with respect to $\left.L_{1}\right)$ we mean that $Q_{\mathrm{u}}(t)$ is defined such that $\left(u_{\mathrm{f}}(t), s(t)\right) \in L_{1}\left(Q_{\mathrm{u}}(t)\right)$ (analogously for $\left.L_{3}\right)$. Since the value of $Q_{\mathrm{u}}$ is uniquely determined by the feed point $\left(u_{\mathrm{f}}, s\right)$, it is convenient to use the notation $Q_{\mathrm{u}}=L_{1}^{-1}\left(u_{\mathrm{f}}, s\right) \Leftrightarrow\left(u_{\mathrm{f}}, s\right) \in L_{1}\left(Q_{\mathrm{u}}\right)$.

To satisfy the three purposes of the settler mentioned above, some control objectives for the process were introduced in [3, Table 1]. The main condition of these is to maintain optimal operation as long as possible. From the analyses of step responses in [2] and dynamic solutions in [4], it turned out to be convenient to introduce the following sets of the operating chart:

$$
\begin{aligned}
\mathcal{S} & =\left\{(u, y): q_{\mathrm{u}} u<y \leq f_{3}(u)\right\}, \\
\mathcal{D} & =\left\{(u, y): 0 \leq u \leq u_{\max }, y>f_{3}(u)\right\}, \\
\mathcal{S}_{1} & =\mathcal{S} \cap\left\{(u, y): y \leq f\left(u_{\text {infl }}\right)\right\}, \\
\mathcal{S}_{2} & =\mathcal{S} \backslash \mathcal{S}_{1},
\end{aligned}
$$

see Figure 3. For step responses from optimal operation in steady state, the state of optimal operation is left immediately if and only if $\left(u_{\mathrm{f}}, s\right) \in \mathcal{D}$. For a general solution, the situation is slightly different. A sufficient condition for maintaining optimal operation, at least for a while, is $\left(u_{\mathrm{f}}, s\right) \in \mathcal{S}_{1}$. 


\subsection{THE CONTROL VARIABLE'S INFLUENCE ON THE UNDERFLOW CONCENTRATION}

Since one of the purposes of the settler is that the underflow concentration should be high, a natural constraint as a part of a control objective is that $u_{\mathrm{u}}(t)$ is bounded below. This can be described in terms of the control variable a priori, see the following theorem (the proof can be found in [3]) and Figure 4.

THEOREM 2.1. Assume that the settler is in optimal operation for $0 \leq t \leq T$.

- The underflow concentration satisfies $u_{\mathrm{u}}(t) \in\left(\overline{\bar{u}}_{\mathrm{u}}, u_{\max }\right]$ for $0 \leq t \leq T$, where $\overline{\bar{u}}_{\mathrm{u}}=$ $f\left(u_{\mathrm{infl}}, \overline{\bar{Q}}_{\mathrm{u}}\right) / \overline{\bar{q}}_{\mathrm{u}}$.

- Let $u_{\mathrm{u}}^{\min } \in\left(\overline{\bar{u}}_{\mathrm{u}}, u_{\max }\right)$ be a given desired lower bound on the underflow concentration. Assume that $Q_{\mathrm{u}}(t) \leq Q_{\mathrm{u}}^{\max 1}$, where $Q_{\mathrm{u}}^{\max 1}$ is defined uniquely by

$$
f\left(u_{\mathrm{M}}\left(Q_{\mathrm{u}}^{\max 1}\right), Q_{\mathrm{u}}^{\max 1}\right)=\frac{Q_{\mathrm{u}}^{\max 1}}{A} u_{\mathrm{u}}^{\min } .
$$

Then $u_{\mathrm{u}}(t) \geq u_{\mathrm{u}}^{\min }$ for $0 \leq t \leq T$ and $Q_{\mathrm{u}}^{\max 1}>\bar{Q}_{\mathrm{u}}$.
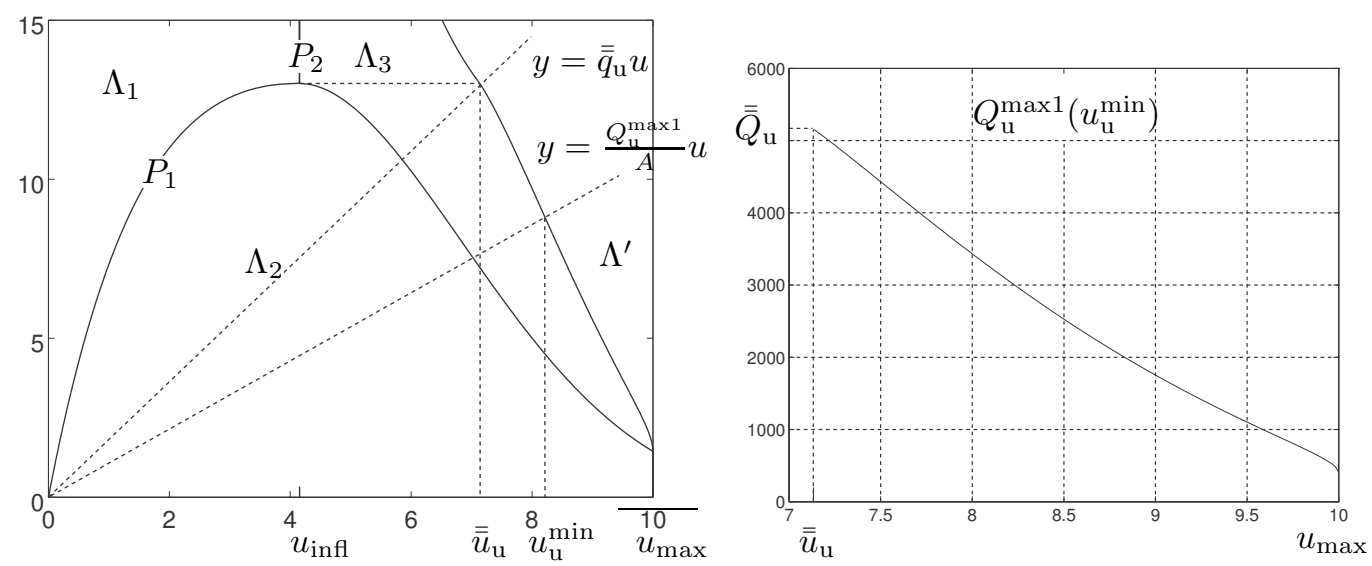

Figure 4. Left: The characteristic concentrations of Theorem 2.1 can be obtained graphically in the operating chart for control of steady states in the following way. $\overline{\bar{u}}_{\mathrm{u}}=7.13 \mathrm{~kg} / \mathrm{m}^{3}$ and $\overline{\bar{Q}}_{\mathrm{u}}=5159 \mathrm{~m}^{3} / \mathrm{h}$ satisfies $f\left(u_{\mathrm{inf}}, \overline{\bar{Q}}_{\mathrm{u}}\right)=\overline{\bar{q}}_{\mathrm{u}} \overline{\bar{u}}_{\mathrm{u}}$. Given $u_{\mathrm{u}}^{\min } \in\left(\overline{\bar{u}}_{\mathrm{u}}, u_{\mathrm{max}}\right)$ determine the corresponding $y$-value on the boundary of $\Lambda_{3}$ and $\Lambda^{\prime}$. This flux value is equal to $Q_{\mathrm{u}}^{\max 1} u_{\mathrm{u}}^{\min } / A$. Right: The graph of $Q_{\mathrm{u}}^{\max 1}$ as a function of $u_{\mathrm{u}}^{\min }$.

\section{Control objectives and strategies}

\subsection{Control objectives}

From the results in [4] we know the limitations of the control variable $Q_{\mathrm{u}}(t)$ for maintaining optimal operation in the sense that overflow is prevented and, if a control objective requires it, the underflow concentration is kept above a prescribed level. However, to maintain optimal operation during a long time, it was also illustrated that there is a need to fine-tune the average SBL so that it stays within the thickening zone. The control objective 'optimal operation is 
maintained as long as possible', possibly subject to a lower bound $u_{\mathrm{u}}^{\min }$ on the underflow concentration, needs therefore to be refined.

Given a fixed reference value $x_{\mathrm{sb}}^{\mathrm{r}}$ of the SBL and a lower bound $u_{\mathrm{u}}^{\mathrm{min}}$, we introduce the following control objectives with respect to the $S B L$ :

COSBL1: Optimal operation is maintained and $x_{\mathrm{sb}}(t)$ is close to $x_{\mathrm{sb}}^{\mathrm{r}}$.

COSBL2: Firstly, $u_{\mathrm{u}}(t) \geq u_{\mathrm{u}}^{\min }$ holds, secondly, optimal operation is maintained and $x_{\mathrm{sb}}(t)$ is close to $x_{\mathrm{sb}}^{\mathrm{r}}$.

The phrase 'close to' could mean, for example, that $x_{\mathrm{sb}}(t)$ lies in an interval around $x_{\mathrm{sb}}^{\mathrm{r}}$. A further natural requirement is that $\left|x_{\mathrm{sb}}(t)-x_{\mathrm{sb}}^{\mathrm{r}}\right|$ should tend to zero if the feed point is constant after a certain time point. Another requirement during periodically varying input data could be that the integrated absolute deviation during a period should be kept small. Then the control variable can be piecewise constant, which could be another constraint.

In order to formulate a control strategy for either of the two control objectives above, the control variable's influence on the SBL as well as the underflow concentration should be known. The latter relation was presented in Section 2.3. The former relation is considered in the next section.

\subsection{DistURBANCES FROM OPTIMAL OPERATION}

For all transient solutions presented in [2,3], it can be concluded that the following property holds: When the settler is in optimal operation, the concentrations above and below the SBL are usually approximately $u_{\mathrm{m}}$ and $u_{\mathrm{M}}$, respectively. This means that a control strategy that succeeds in meeting COSBL1 or 2, yields a dynamic solution that is approximately a stationary optimal-operation solution. Therefore, to elaborate such a control strategy, it is of vital importance to have information of the responses of the process to disturbances when the settler is in optimal operation in steady state.

The relation between $Q_{\mathrm{u}}(t)$ and $x_{\mathrm{sb}}(t)$ is difficult to obtain generally. In fact, there is no unique relation, since the actual concentration distribution in the settler plays a role. From [1] we know that given that the triple $\left(u_{\mathrm{f}}, s, Q_{\mathrm{u}}\right)$ implies a steady-state solution in optimal operation, the solution is unique except for the location of the SBL, which can be anywhere in the thickening zone. Hence, the only relation in such a case is that $x_{\mathrm{sb}}$ is constant as long as $Q_{\mathrm{u}}$ is (and the feed point is constant). However, given the location of $x_{\mathrm{sb}}$ we can establish the response to a change in $Q_{\mathrm{u}}$.

Assume that the settler is in optimal operation in steady state. Then the feed point $\left(u_{\mathrm{f} 0}, s_{0}\right)$ lies on the horizontal straight part of $L_{1}\left(Q_{\mathrm{u} 0}\right)$, see Figure 3. We investigate four disturbances of this state such that the feed point ends up above or below the horizontal line. We are only interested in small disturbances such that optimal operation is not left directly.

A feed-point step such that $\left(u_{\mathrm{f}}, s\right)$ lies below $L_{1}\left(Q_{\mathrm{u} 0}\right)$

After a step change in the feed point to $\left(u_{\mathrm{f}}, s\right) \in \mathcal{U}_{1}\left(Q_{\mathrm{u} 0}\right)$, which means that $s<s_{0}$, the SBL is constant for a while and then declines. The mass decreases (linearly). This was shown in [2, Section 4: case $\left.\mathcal{U}_{1}\right]$.

A control-variable step up such that $\left(u_{\mathrm{f} 0}, s_{0}\right)$ lies below $L_{1}\left(Q_{\mathrm{u}}\right)$

As the control parameter jumps up to $Q_{\mathrm{u}}>Q_{\mathrm{u} 0}$ such that $\left(u_{\mathrm{f} 0}, s_{0}\right) \in \mathcal{U}_{1}\left(Q_{\mathrm{u}}\right)$ holds, the solution in the thickening zone is qualitatively as the one constructed in Figure 5. A simulation is 
shown in Figure 6. We can conclude that the SBL declines, i.e. $x_{\mathrm{sb}}(t)$ increases. The incoming
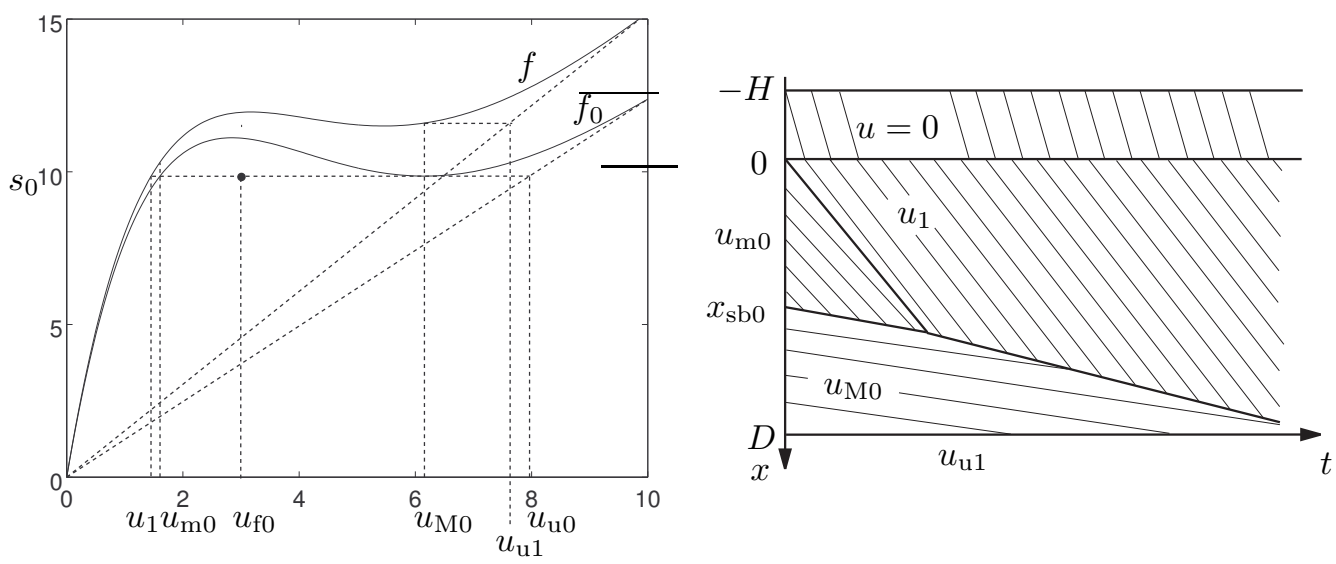

Figure 5. The case when $\left(u_{\mathrm{f} 0}, s_{0}\right)$ lies below $L_{1}\left(Q_{\mathrm{u}}\right)$ after a step up of the control variable. The flux functions (left) are $f_{0}(u)=f\left(u, Q_{\mathrm{u} 0}\right)$ and $f(u)=f\left(u, Q_{\mathrm{u}}\right)$. The solution (right) consists of three different concentrations in the thickening zone, separated by discontinuities. Thin lines are characteristics.
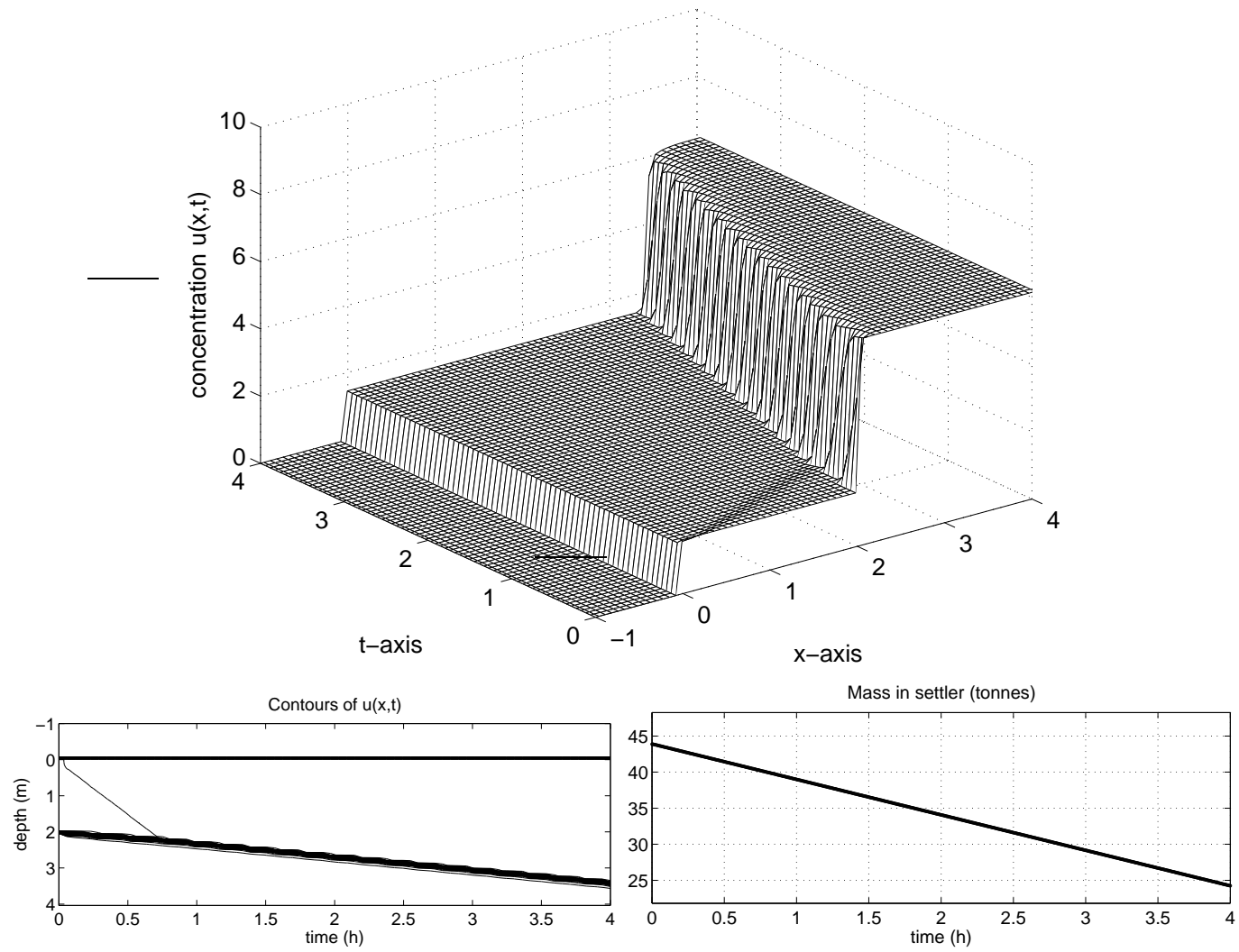

Figure 6. A simulation in the case when $\left(u_{\mathrm{f} 0}, s_{0}\right)=(3,11.5)$ lies below $L_{1}\left(Q_{\mathrm{u}}\right)$ after a step up from $Q_{\mathrm{u} 0}=3500$ to $Q_{\mathrm{u}}=4298$. The underflow concentration steps down from $u_{\mathrm{u} 0}=7.96$ to $u_{\mathrm{u}}(t)=6.89$ for $0<t<4$.

mass per time unit, $Q_{\mathrm{f} 0} u_{\mathrm{f} 0}=A s_{0}=Q_{\mathrm{u} 0} u_{\mathrm{u} 0}$, is unchanged. Despite the fact that $u_{\mathrm{u} 1}<u_{\mathrm{u} 0}$, the outgoing mass per time unit, $Q_{\mathrm{u}} u_{\mathrm{u} 1}$ is greater than $Q_{\mathrm{u} 0} u_{\mathrm{u} 0}$, since the mass decreases. The 
latter follows from the fact that for each $x \in(0, D)$ the concentration is non-increasing with time.

A feed-point step such that $\left(u_{\mathrm{f}}, s\right)$ lies above $L_{1}\left(Q_{\mathrm{u} 0}\right)$

From the step responses in [2, Section 4: cases $\left.\mathcal{O}_{2 \mathrm{a}}, \mathcal{O}_{3 \mathrm{a}}, \ell_{4 \mathrm{a}}, \mathcal{U}_{2 \mathrm{a}}\right]$, in which $s>s_{0}$, it can be concluded that the SBL is constant for a while and then rises. The mass increases (linearly).

A control-variable step down such that $\left(u_{\mathrm{f} 0}, s_{0}\right)$ lies above $L_{1}\left(Q_{\mathrm{u}}\right)$

As the control parameter jumps down to $Q_{\mathrm{u}}<Q_{\mathrm{u} 0}$ such that $\left(u_{\mathrm{f} 0}, s_{0}\right)$ belongs to the region between the lines $L_{1}$ and $L_{3}$ in Figure 3. The solution in the thickening zone is qualitatively as the one constructed in Figure 7. A simulation is shown in Figure 8. We can conclude that the
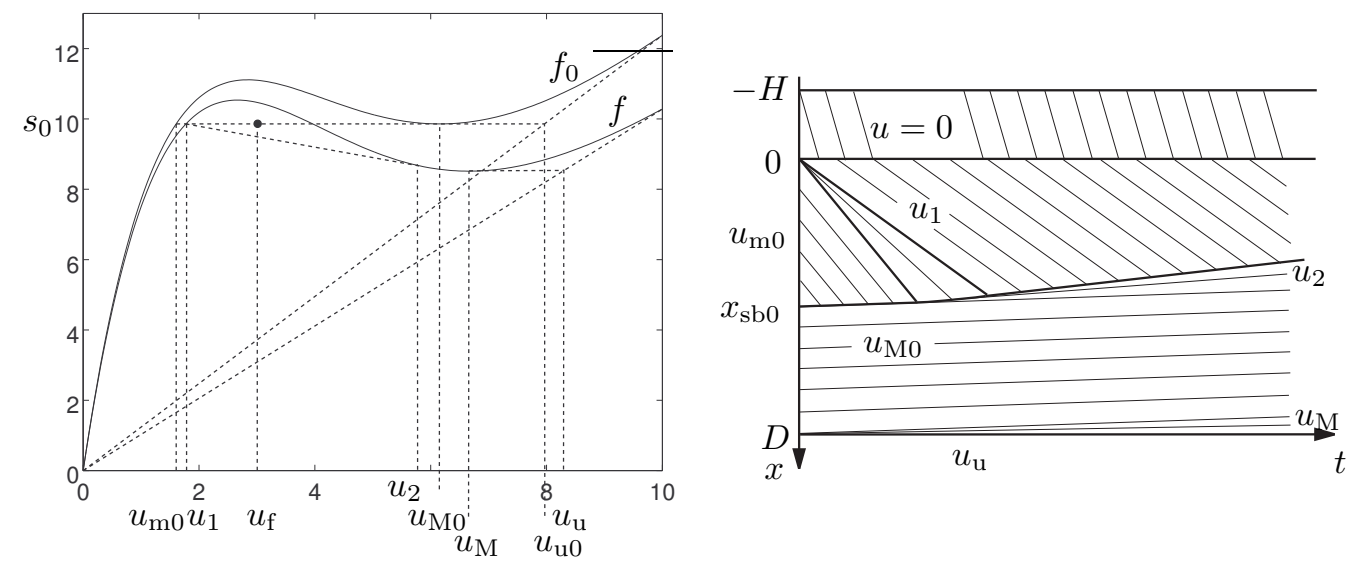

Figure 7. The case when $\left(u_{\mathrm{fo}}, s_{0}\right)$ lies above $L_{1}\left(Q_{\mathrm{u}}\right)$ after a step down in the control variable. Flux functions (left) and the solution (right). The bottom concentration is $u_{\mathrm{M}}$ for $t>0$. The SBL rises, first with a constant speed, then with an increasing speed (as the SBL is a contact discontinuity), and then with a constant speed.

SBL rises, i.e. $x_{\mathrm{sb}}(t)$ decreases. The incoming mass per time unit, $Q_{\mathrm{f} 0} u_{\mathrm{f} 0}=A s_{0}=Q_{\mathrm{u} 0} u_{\mathrm{u} 0}$, is unchanged. Despite the fact that $u_{\mathrm{u} 1}>u_{\mathrm{u} 0}$, the outgoing mass per time unit, $Q_{\mathrm{u}} u_{\mathrm{u} 1}$ is less than $Q_{\mathrm{u} 0} u_{\mathrm{u} 0}$, since the mass increases. This follows from the fact that for each $x \in(0, D)$ the concentration is non-decreasing with time.

\section{A fundamental property}

All four cases above with small step-disturbances of a solution in optimal operation show the following fundamental property, which is well known among all operators of clarifierthickeners (e.g. [48]):

$$
\begin{aligned}
& Q_{\mathrm{u}}(t) \text { decreases or } s(t) \text { increases } \quad \Longrightarrow \quad m(t) \text { increases and the SBL rises, } \\
& Q_{\mathrm{u}}(t) \text { increases or } s(t) \text { decreases } \Longrightarrow m(t) \text { decreases and the SBL declines. }
\end{aligned}
$$

A control strategy must take this fundamental property into account. We can note from the four cases that the influence on the mass is direct, whereas there may be a time delay before the SBL changes.

Furthermore, the SBL may be difficult to measure in a plant, particularly during transients. Since the concentrations and flows of the input and output streams can be measured, the total mass in the settler can be calculated. Our control strategy will be to control the total mass by 


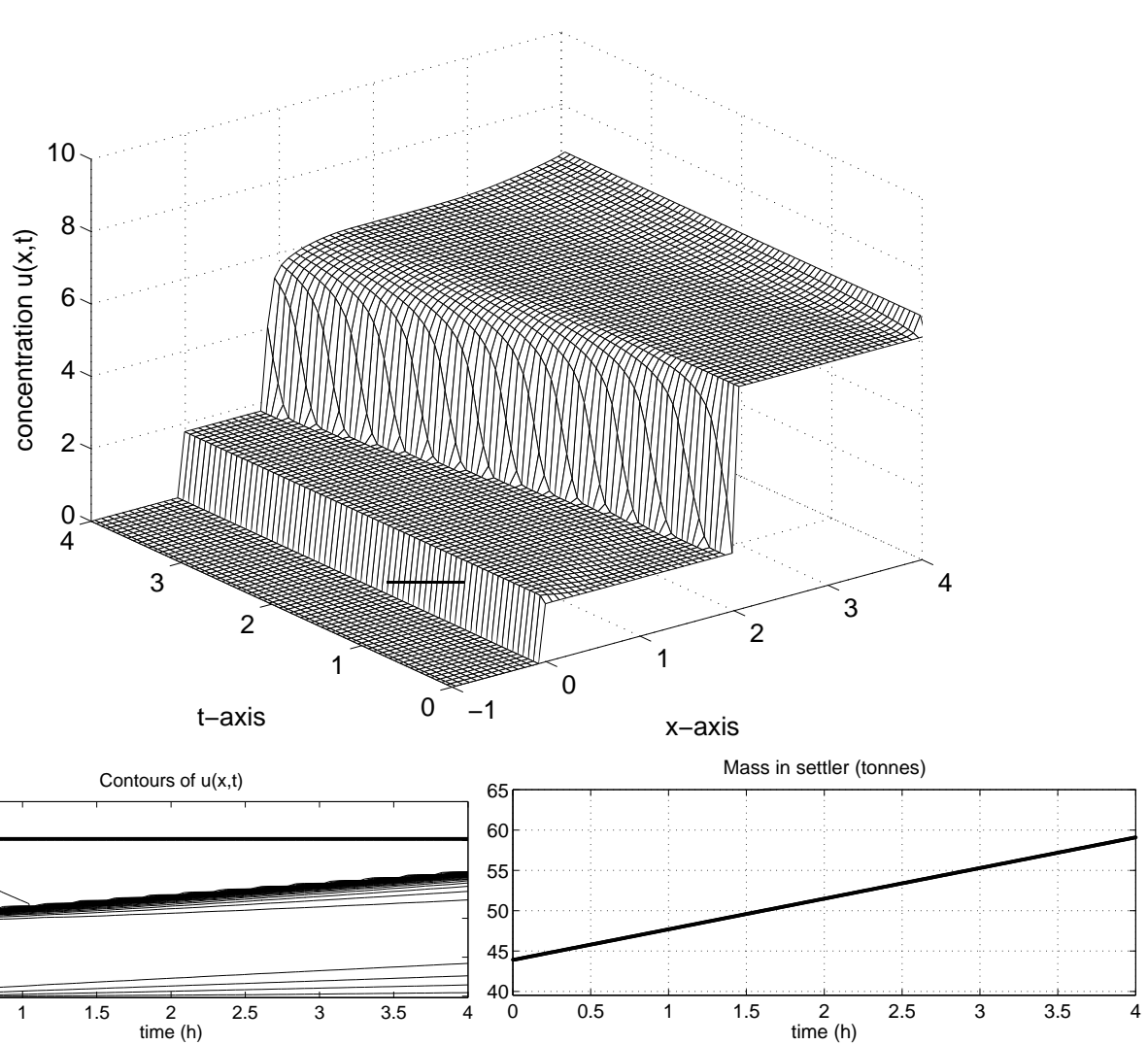

Figure 8. A simulation in the case when $\left(u_{\mathrm{fo}}, s_{0}\right)=(3,11.5)$ lies above $L_{1}\left(Q_{\mathrm{u}}\right)$ after a step down from $Q_{\mathrm{u} 0}=3500$ to $Q_{\mathrm{u}}=2907$. The underflow concentration steps up from $u_{\mathrm{u} 0}=7.96$ to $u_{\mathrm{u}}(t)=9.69$ for $t>0$.

using a regulator. To ensure that such a regulator satisfies the control objectives, we need a relation between the mass and the SBL.

\subsection{The STEAdy-STATE RELATION BETWEen the MASS, THE SBL AND THE CONTROL VARIABLE}

For optimal operation in steady state the following relation holds between the mass, the SBL and the control variable:

$$
\begin{aligned}
m_{\mathrm{ss}}\left(x_{\mathrm{sb}}, Q_{\mathrm{u}}\right) & =A\left(x_{\mathrm{sb}} u_{\mathrm{m}}\left(Q_{\mathrm{u}}\right)+\left(D-x_{\mathrm{sb}}\right) u_{\mathrm{M}}\left(Q_{\mathrm{u}}\right)\right)= \\
& =A\left(D u_{\mathrm{M}}\left(Q_{\mathrm{u}}\right)-x_{\mathrm{sb}}\left(u_{\mathrm{M}}\left(Q_{\mathrm{u}}\right)-u_{\mathrm{m}}\left(Q_{\mathrm{u}}\right)\right)\right),
\end{aligned}
$$

where $Q_{\mathrm{u}}=L_{1}^{-1}\left(u_{\mathrm{f}}, s\right)$. A three-dimensional graph of this function is shown in Figure 9. Note that, for fixed $Q_{\mathrm{u}}, m$ depends affinely on $x_{\mathrm{sb}}$. From [1] we know that given that the triple $\left(u_{\mathrm{f}}, s, Q_{\mathrm{u}}\right)$ implies a steady-state solution in optimal operation, it is unique except for the location of the SBL, which can be anywhere in the thickening zone. This implies that there is no relation between $Q_{\mathrm{u}}$ and $x_{\mathrm{sb}}$ in (2).

Another interesting thing regarding the control problem is the following. For fixed $x_{\mathrm{sb}}$, the mass is a weighted average of the two concentrations $u_{\mathrm{m}}$ and $u_{\mathrm{M}}$. Especially, as the SBL is in 


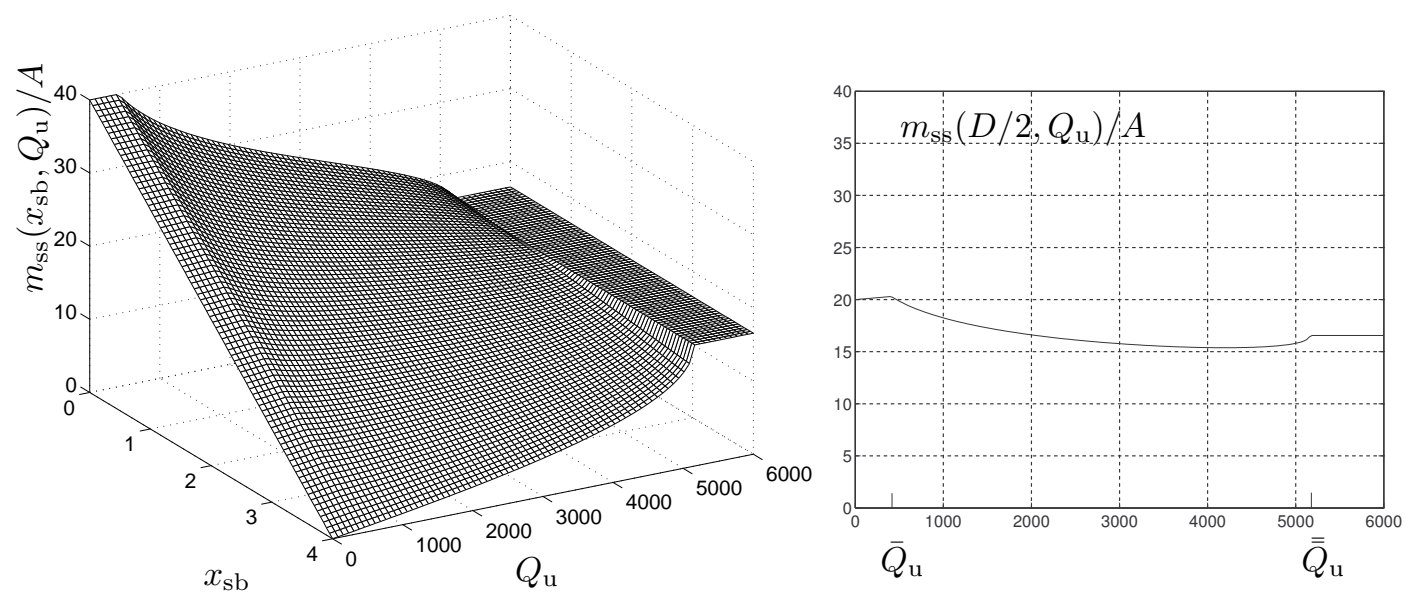

Figure 9. Left: The relation between the mass in the settler (normalized by $A$ ), the SBL and the control variable when the settler is in optimal operation in steady state. This function depends on the batch settling flux $f_{\mathrm{b}}$. Recall that $D=4 \mathrm{~m}$. Right: The relation as $x_{\mathrm{sb}}=D / 2=2 \mathrm{~m}$.

the middle of the thickening zone, $x_{\mathrm{sb}}=D / 2$, the weights are equal and

$$
m_{\mathrm{ss}}\left(D / 2, Q_{\mathrm{u}}\right)=A D \frac{u_{\mathrm{m}}\left(Q_{\mathrm{u}}\right)+u_{\mathrm{M}}\left(Q_{\mathrm{u}}\right)}{2} .
$$

Since $u_{\mathrm{m}}\left(Q_{\mathrm{u}}\right) \nearrow u_{\mathrm{infl}}$ and $u_{\mathrm{M}}\left(Q_{\mathrm{u}}\right) \searrow u_{\mathrm{infl}}$ as $Q_{\mathrm{u}} \nearrow \bar{Q}_{\mathrm{u}}, m_{\mathrm{ss}}\left(D / 2, Q_{\mathrm{u}}\right)$ is approximately constant for the given batch flux function, see Figure 9 (right).

During dynamic operation (2) does not hold. However, $m_{\mathrm{ss}}\left(x_{\mathrm{sb}}(t), Q_{\mathrm{u}}(t)\right)$ seems to be a fairly good approximation of the mass $m(t)$, because of the above-mentioned property that a controlled settler in optimal operation is approximately in optimal operation in steady state.

\subsection{A CONTROL STRATEGY}

From [3, Table 2] we can conclude that a necessary condition for keeping optimal operation after a step input is that $\left(u_{\mathrm{f}}, s\right) \in P_{1} \cup \Lambda_{2} \cup \Lambda_{3 \mathrm{a}}$, see Figure 2. If, in addition, the SBL is not too close to the bottom (inequality (9) in [3] holds), optimal operation can be maintained. Furthermore, if the SBL meets the bottom, it was shown that the SBL can be restored within the thickening zone again after a finite time.

Accordingly, a necessary condition for maintaining optimal operation during long time of dynamic operation is that

$$
\left(u_{\mathrm{f}}(t), s(t)\right) \in P_{1} \cup \Lambda_{2} \cup \Lambda_{3 \mathrm{a}} .
$$

Assuming this holds we define the reference value of the control parameter either as

$$
Q_{\mathrm{u}}^{\mathrm{r}}(t)=L_{1}^{-1}\left(u_{\mathrm{f}}(t), s(t)\right) \quad \Longleftrightarrow s(t)=f\left(u_{\mathrm{M}}\left(Q_{\mathrm{u}}^{\mathrm{r}}(t)\right), Q_{\mathrm{u}}^{\mathrm{r}}(t)\right)
$$

or

$$
Q_{\mathrm{u}}^{\mathrm{r}}(t)=L_{1}^{-1}\left(u_{\mathrm{f}}^{\mathrm{av}}(t), s^{\mathrm{av}}(t)\right), \quad \text { where } \quad u_{\mathrm{f}}^{\mathrm{av}}(t)=\int_{t}^{t+T} u_{\mathrm{f}}(\tau) d \tau
$$


(analogously for $s^{\mathrm{av}}$ ) for some positive number $T$, preferably the period in the case of a periodic input. Guided by (2) we then define the reference mass:

$$
m^{\mathrm{r}}(t)=m_{\mathrm{ss}}\left(x_{\mathrm{sb}}^{\mathrm{r}}, Q_{\mathrm{u}}^{\mathrm{r}}(t)\right) .
$$

Because of the two relationships (2) and (6) the absolute difference $\left|x_{\mathrm{sb}}(t)-x_{\mathrm{sb}}^{\mathrm{r}}\right|$ is small if and only if $\left|m_{\mathrm{ss}}\left(x_{\mathrm{sb}}(t), Q_{\mathrm{u}}^{\mathrm{r}}(t)\right)-m^{\mathrm{r}}(t)\right|$ is small. Combining this with the abovedescribed property that $m(t)$ is approximated by $m_{\mathrm{ss}}\left(x_{\mathrm{sb}}(t), Q_{\mathrm{u}}^{\mathrm{r}}(t)\right)$ in optimal operation, we conclude that a control strategy should keep $\left|m(t)-m^{\mathrm{r}}(t)\right|$ small. The fundamental property of Section 3.2 yields the first part of the following control strategy: define $Q_{\mathrm{u}}(t)$ such that

- $Q_{\mathrm{u}}(t)-Q_{\mathrm{u}}^{\mathrm{r}}(t)=h\left(m(t)-m^{\mathrm{r}}(t)\right)$ for some increasing function $h$ with $h(0)=0$.

- optimal operation is maintained, and, for COSBL2, the underflow concentration is bounded below.

The first item is achieved by a proportional regulator, see the next section. The second item is achieved by adding saturating bounds, which are obtained from the results in [4] and presented below in Section 5.

\section{A proportional regulator}

\subsection{THE REgUlator}

The first item of the control strategy above is in this section implemented in terms of a proportional regulator. Assume that optimal operation holds for $t>0$.

Given the initial mass $m_{0}$ in the settler at $t=0$, the mass at time $t$ is given by

$$
m(t)=m_{0}+\int_{0}^{t}\left(Q_{\mathrm{f}}(t) u_{\mathrm{f}}(t)-Q_{\mathrm{u}}(t) u_{\mathrm{u}}(t)\right) d t, \quad t>0,
$$

which is equivalent to

$$
\left\{\begin{array}{l}
\frac{d m}{d t}=A s(t)-Q_{\mathrm{u}}(t) u_{\mathrm{u}}(t), \quad t>0, \\
m(0)=m_{0}
\end{array}\right.
$$

Note that $m(t)$ is continuous and piecewise differentiable, since $s, Q_{\mathrm{u}}$ and $u_{\mathrm{u}}$ are piecewise $C^{1}$. For a constant $K>0$ we introduce the proportional regulator

$$
Q_{\mathrm{u}}(t)=Q_{\mathrm{u}}^{\mathrm{r}}(t)+K\left(m(t)-m^{\mathrm{r}}(t)\right) .
$$

Substituting (9) into (8) yields the linear, time-varying equation

$$
\left\{\begin{array}{l}
\frac{d m}{d t}+K u_{\mathrm{u}}(t)\left(m(t)-m^{\mathrm{r}}(t)\right)=A s(t)-Q_{\mathrm{u}}^{\mathrm{r}}(t) u_{\mathrm{u}}(t), \quad t>0, \\
m(0)=m_{0} .
\end{array}\right.
$$

This can be integrated to 


$$
\begin{aligned}
m(t) & =m_{0} e^{-K U_{\mathrm{u}}(t)}+ \\
& +\int_{0}^{t}\left(K u_{\mathrm{u}}(\tau) m^{\mathrm{r}}(\tau)+A s(\tau)-Q_{\mathrm{u}}^{\mathrm{r}}(\tau) u_{\mathrm{u}}(\tau)\right) e^{-K\left(U_{\mathrm{u}}(t)-U_{\mathrm{u}}(\tau)\right)} d \tau, \quad t>0,
\end{aligned}
$$

where

$$
U_{\mathrm{u}}(t)=\int_{0}^{t} u_{\mathrm{u}}(\tau) d \tau
$$

Since optimal operation is assumed to hold, Theorem 2.1 implies $\overline{\bar{u}}_{\mathrm{u}}<u_{\mathrm{u}}(t) \leq u_{\max }$, and we have the bounds

$$
0<\overline{\bar{u}}_{\mathrm{u}} t<U_{\mathrm{u}}(t) \leq u_{\max } t \text { for } t>0 .
$$

Hence, the first term on the right-hand side of (10) tends to zero exponentially as $t \rightarrow \infty$.

Assume now that the feed point remains constant after a certain time as well as the reference value $Q_{\mathrm{u}}^{\mathrm{r}}$, which may or may not be chosen according to (4). Then the reference mass $m^{\mathrm{r}}$, defined by (6), is also constant. With the constant $u_{\mathrm{u}}^{\mathrm{r}} \equiv A s / Q_{\mathrm{u}}^{\mathrm{r}},(10)$ can be reduced to

$$
m(t)=m^{\mathrm{r}}+\left(m_{0}-m^{\mathrm{r}}\right) e^{-K U_{\mathrm{u}}(t)}+Q_{\mathrm{u}}^{\mathrm{r}} \int_{0}^{t}\left(u_{\mathrm{u}}^{\mathrm{r}}-u_{\mathrm{u}}(\tau)\right) e^{-K\left(U_{\mathrm{u}}(t)-U_{\mathrm{u}}(\tau)\right)} d \tau .
$$

The second term on the right-hand side tends to zero exponentially by (11). Assuming that the solution converges to a stationary solution in optimal operation then the last term in (12) tends to a constant, which is zero in the case $Q_{\mathrm{u}}^{\mathrm{r}}$ is defined by (4), as $t \rightarrow \infty$. This can be proved in the following way. For given $\left(u_{\mathrm{f}}, s\right)$ the value of the control parameter for a corresponding solution in optimal operation is unique, see [1, Theorem 2]: $Q_{\mathrm{u}}(\infty) \equiv \lim _{t \rightarrow \infty} Q_{\mathrm{u}}(t)=$ $L_{1}^{-1}\left(u_{\mathrm{f}}, s\right)$. Hence, (9) implies that

$$
m(\infty)=m^{\mathrm{r}}+\frac{1}{K}\left(Q_{\mathrm{u}}(\infty)-Q_{\mathrm{u}}^{\mathrm{r}}\right) .
$$

Furthermore, for the stationary solution in the limit, (2) yields

$$
m(\infty)=A\left(D u_{\mathrm{M}}\left(Q_{\mathrm{u}}(\infty)\right)-x_{\mathrm{sb}}(\infty)\left(u_{\mathrm{M}}\left(Q_{\mathrm{u}}(\infty)\right)-u_{\mathrm{m}}\left(Q_{\mathrm{u}}(\infty)\right)\right)\right)
$$

from which $x_{\mathrm{sb}}(\infty)$ can be calculated. Combined with (13) we get

$$
x_{\mathrm{sb}}(\infty)=\frac{D u_{\mathrm{M}}\left(Q_{\mathrm{u}}(\infty)\right)-\frac{1}{A} m(\infty)}{u_{\mathrm{M}}\left(Q_{\mathrm{u}}(\infty)\right)-u_{\mathrm{m}}\left(Q_{\mathrm{u}}(\infty)\right)}=\frac{D u_{\mathrm{M}}\left(Q_{\mathrm{u}}(\infty)\right)-\frac{1}{A}\left(m^{\mathrm{r}}+\frac{1}{K}\left(Q_{\mathrm{u}}(\infty)-Q_{\mathrm{u}}^{\mathrm{r}}\right)\right)}{u_{\mathrm{M}}\left(Q_{\mathrm{u}}(\infty)\right)-u_{\mathrm{m}}\left(Q_{\mathrm{u}}(\infty)\right)} .
$$

If, and only if, (4) is used to define the reference value $Q_{\mathrm{u}}^{\mathrm{r}}=L_{1}^{-1}\left(u_{\mathrm{f}}, s\right)=Q_{\mathrm{u}}(\infty)$, (13) implies $m(\infty)=m^{\mathrm{r}}$ and (14) becomes

$$
m^{\mathrm{r}}=A\left(D u_{\mathrm{M}}\left(Q_{\mathrm{u}}^{\mathrm{r}}\right)-x_{\mathrm{sb}}(\infty)\left(u_{\mathrm{M}}\left(Q_{\mathrm{u}}^{\mathrm{r}}\right)-u_{\mathrm{m}}\left(Q_{\mathrm{u}}^{\mathrm{r}}\right)\right)\right) .
$$

This affine relationship between $m^{\mathrm{r}}$ and $x_{\mathrm{sb}}(\infty)$, together with the corresponding one (6) between $m^{\mathrm{r}}$ and $x_{\mathrm{sb}}^{\mathrm{r}}$, yields $x_{\mathrm{sb}}(\infty)=x_{\mathrm{sb}}^{\mathrm{r}}$. We sum up the results. 
THEOREM 4.1. Given $x_{\mathrm{sb}}^{\mathrm{r}} \in(0, D)$, arbitrary initial data and a constant feed point $\left(u_{\mathrm{f}}, s\right)$ for $t>0$. Assume that the regulator (9), with a given constant $Q_{\mathrm{u}}^{\mathrm{r}}$, connected with (1) implies that the solution is in optimal operation and converges to a stationary solution as $t \rightarrow \infty$. Then the limit $S B L$ is given by $(15)$, with $Q_{\mathrm{u}}(\infty)=L_{1}^{-1}\left(u_{\mathrm{f}}, s\right)$. In particular, if $Q_{\mathrm{u}}^{\mathrm{r}}=L_{1}^{-1}\left(u_{\mathrm{f}}, s\right)$ then $x_{\mathrm{sb}}(t) \rightarrow x_{\mathrm{sb}}^{\mathrm{r}}$ as $t \rightarrow \infty$.

Remark 1. The rate of convergence to zero of the second term of (12) is exponential. In the case $Q_{\mathrm{u}}^{\mathrm{r}}=L_{1}^{-1}\left(u_{\mathrm{f}}, s\right)$ it follows from the theorem and (12) that $u_{\mathrm{u}}(t) \rightarrow u_{\mathrm{u}}^{\mathrm{r}}$ as $t \rightarrow \infty$. The rate of convergence of this limit process is difficult to establish since it depends on the solution within the settler, which in turn depends on the control parameter value from the regulator (9). Hence, the rate of convergence of the third term of (12) is difficult to obtain, and therefore likewise for the limit process $x_{\mathrm{sb}}(t) \rightarrow x_{\mathrm{sb}}^{\mathrm{r}}$.

Remark 2. Independently of $K, Q_{\mathrm{u}}(t) \rightarrow L_{1}^{-1}\left(u_{\mathrm{f}}, s\right)$ as $t \rightarrow \infty$. Note that (13) implies that $m(\infty) \rightarrow m^{\mathrm{r}}$ as $K \rightarrow \infty$ independently of whether $Q_{\mathrm{u}}^{\mathrm{r}}$ is chosen equal to $L_{1}^{-1}\left(u_{\mathrm{f}}, s\right)=$ $Q_{\mathrm{u}}(\infty)$ or not. If $Q_{\mathrm{u}}^{\mathrm{r}} \neq Q_{\mathrm{u}}(\infty)$, then $x_{\mathrm{sb}}(\infty) \nrightarrow x_{\mathrm{sb}}^{\mathrm{r}}$ holds generally as $K \rightarrow \infty$, which can be inferred from (15) and (6):

$$
\begin{aligned}
x_{\mathrm{sb}}(\infty) & \rightarrow \frac{D u_{\mathrm{M}}\left(Q_{\mathrm{u}}(\infty)\right)-\frac{1}{A} m^{\mathrm{r}}}{u_{\mathrm{M}}\left(Q_{\mathrm{u}}(\infty)\right)-u_{\mathrm{m}}\left(Q_{\mathrm{u}}(\infty)\right)}, \quad K \rightarrow \infty, \\
x_{\mathrm{sb}}^{\mathrm{r}} & =\frac{D u_{\mathrm{M}}\left(Q_{\mathrm{u}}^{\mathrm{r}}\right)-\frac{1}{A} m^{\mathrm{r}}}{u_{\mathrm{M}}\left(Q_{\mathrm{u}}^{\mathrm{r}}\right)-u_{\mathrm{m}}\left(Q_{\mathrm{u}}^{\mathrm{r}}\right)} .
\end{aligned}
$$

\subsection{SOME PROPERTIES OF THE PROPORTIONAL REGULATOR}

We demonstrate the statements of Theorem 4.1 by considering a numerical example. Initially, optimal operation holds with the SBL in the middle of the thickening zone; $x_{\mathrm{sb} 0}=D / 2=$ $2 \mathrm{~m}$. The feed point is $\left(u_{\mathrm{f} 0}, s_{0}\right)=\left(3 \mathrm{~kg} / \mathrm{m}^{3}, 9.86 \mathrm{~kg} /\left(\mathrm{m}^{2} \mathrm{~h}\right)\right)$ and $Q_{\mathrm{u} 0}=3500 \mathrm{~m}^{3} / \mathrm{h}$. At $t=0$ there is a step change to $\left(u_{\mathrm{f}}, s\right)=(3,11.5) \in \mathcal{O}\left(Q_{\mathrm{u} 0}\right) \cap \Lambda_{2}$. Without any change in the control variable, there will be a rising SBL and an overloaded settler after a finite time, see Figure 10. When DCL1 is used to define the new constant value $Q_{\mathrm{u}}=4298$ for $t>0$, the response (without a regulator) is shown in [3, Figures 17-18]. Optimal operation is maintained, but the new SBL satisfies $x_{\mathrm{sb}}(\infty)>x_{\mathrm{sb} 0}$.

Connecting the regulator (9) with $K=1 \mathrm{~m}^{3} /(\mathrm{kgh}), x_{\mathrm{sb}}^{\mathrm{r}}=2 \mathrm{~m}$ and $Q_{\mathrm{u}}^{\mathrm{r}}=L_{1}^{-1}(3,11.5)=$ 4298 , the original SBL is restored, see the simulation result in Figure 11.

A higher value of $K$ gives a more rapid convergence of $Q_{\mathrm{u}}(t)$ and $m(t)$. However, there is a transient solution in which the SBL may not converge much faster. We demonstrate this by setting $K=10$ in Figure 12 .

Even if $Q_{\mathrm{u}}^{\mathrm{r}}$ is not defined according to (4) the regulator may be of major importance to maintain optimal operation, cf. Theorem 4.1. Assume that the regulator (9) is connected with the constant value $Q_{\mathrm{u}}^{\mathrm{r}}=Q_{\mathrm{u} 0}=3500$. Then $Q_{\mathrm{u}}(t)$ is continuous, and hence also $u_{\mathrm{u}}(t)$. The simulation with $K=1$ in Figure 13 shows a similar behaviour as in Figure 11 with the difference that the mass now converges to a slightly higher value. According to Theorem 4.1, $Q_{\mathrm{u}}(t) \rightarrow L_{1}^{-1}(3,11.5)=4298$ as $t \rightarrow \infty$. Formulae (13) and (15) yield $m(\infty)=44.7$ tonnes and $x_{\mathrm{sb}}(\infty)=1.87 \mathrm{~m}$, respectively. Note that these two values depend on the regulator gain 

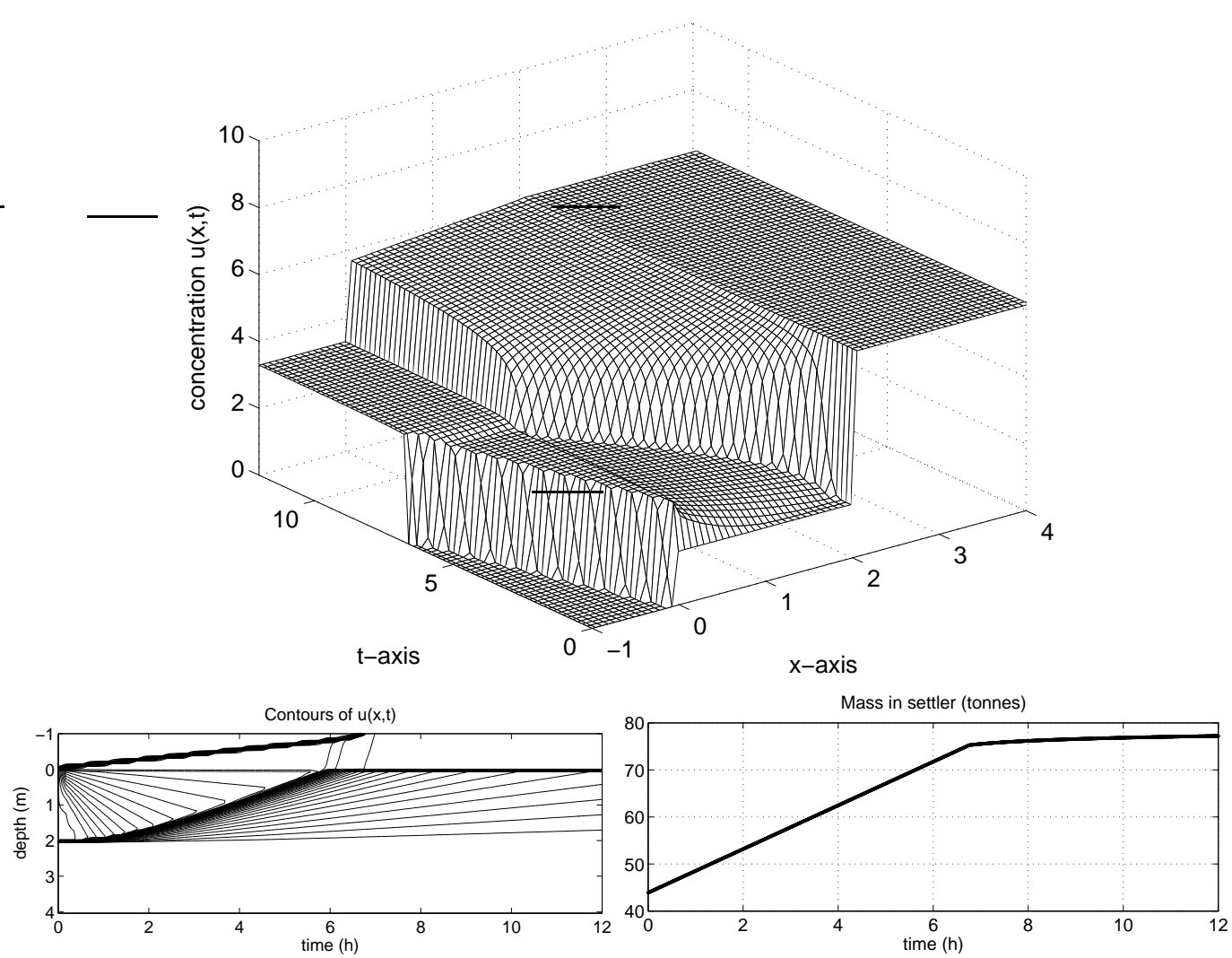

Figure 10. A simulation of a step response resulting in an overloaded settler when no regulator is connected $\left(Q_{\mathrm{u}}(t)=Q_{\mathrm{u} 0}=3500 \mathrm{~m}^{3} / \mathrm{h}\right)$.

$K$. If $K$ is chosen to a larger or smaller value instead, the regulator implies different limit values of the mass and the SBL, see Figures 14 and 15.
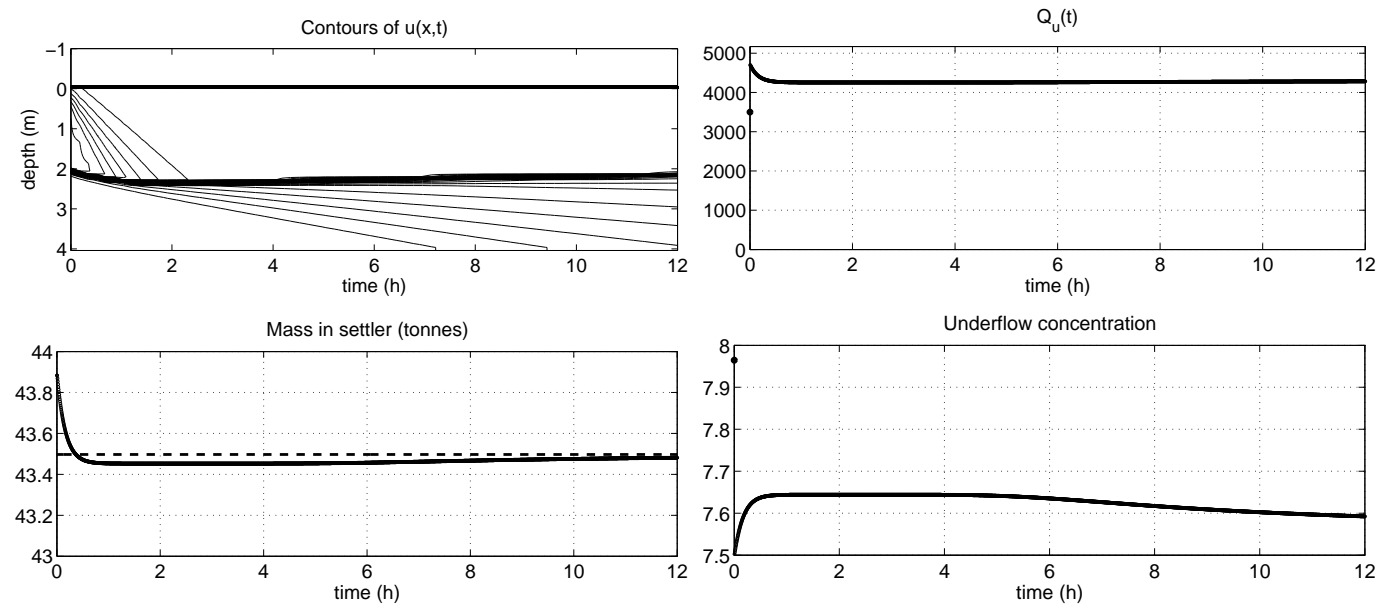

Figure 11. A numerical simulation during 12 hours of a step change to $\left(u_{\mathrm{f}}, s\right)=(3,11.5) \in \mathcal{O}\left(Q_{\mathrm{u} 0}\right) \cap \Lambda_{2}$ when the regulator (9) is connected with $K=1, x_{\mathrm{sb}}^{\mathrm{r}}=2 \mathrm{~m}$ and $Q_{\mathrm{u}}^{\mathrm{r}}=L_{1}^{-1}(3,11.5)=4298 \mathrm{~m}^{3} / \mathrm{h}$. The constant reference mass $m^{\mathrm{r}}(t)=43.5$ tonnes, given by (6), is shown by the dashed line. The regulator restores the initial $\mathrm{SBL}$, and the mass and control variable converge to its reference values. 

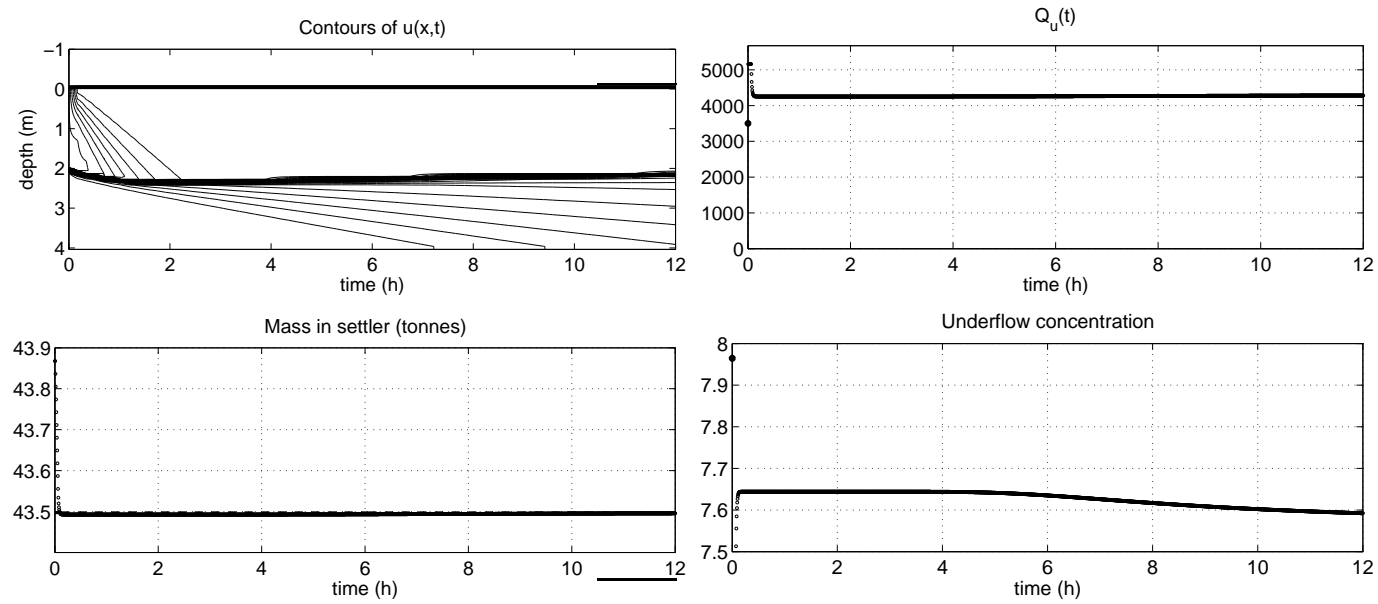

Figure 12. A simulation using the same data as in Figure 11 but with $K=10$ instead. $Q_{\mathrm{u}}(t)$ and $m(t)$ converge faster, but not $x_{\mathrm{sb}}(t)$.
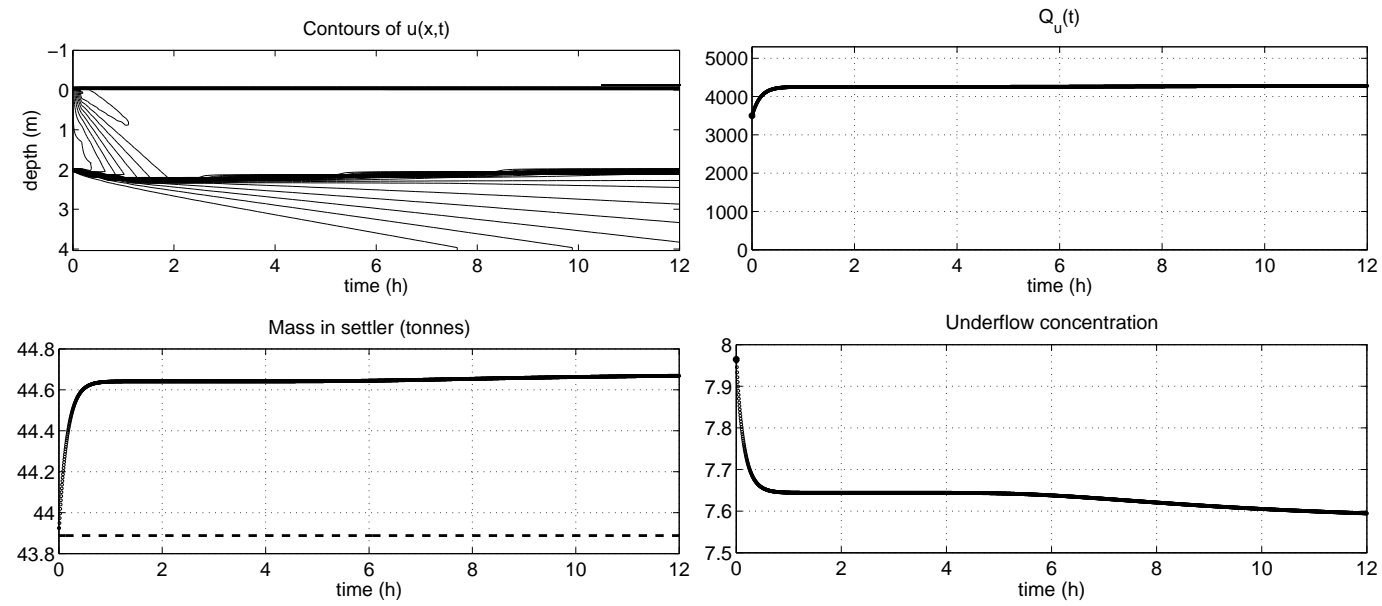

Figure 13. A simulation using the same data as in Figure $11(K=1)$, but with $Q_{\mathrm{u}}^{\mathrm{r}}=Q_{\mathrm{u} 0}=3500$ instead. The regulator implies that $m(t) \rightarrow 44.7$ tonnes and $x_{\mathrm{sb}}(t) \rightarrow 1.87 \mathrm{~m}$ as $t \rightarrow \infty$.

Because of the feed point jump to $(3,11.5) \in \mathcal{D}\left(Q_{\mathrm{u} 0}\right)$, and the fact that $Q_{\mathrm{u}}(t)$ is continuous, the settler is actually overloaded for small $t>0$ with some particles in the clarification zone. With the resolution of the simulations, this can only be hinted in the contour graph in Figure 15, where the small $K$ means a sluggish $Q_{\mathrm{u}}(t)$. The condition $(3,11.5) \in \mathcal{D}\left(Q_{\mathrm{u}}(t)\right)$ holds during the time when $Q_{\mathrm{u}}(t)<L_{3}^{-1}(3,11.5)=3876$, which is less than an hour in Figure 15.

Compare the simulation in Figure 14 with the second remark after Theorem 4.1. The simulation shows that the high value $K=10$ implies $m(\infty) \approx m^{\mathrm{r}}$ in accordance with $m(\infty) \rightarrow m^{\mathrm{r}}$ as $K \rightarrow \infty$. The simulation also shows that $x_{\mathrm{sb}}(t)$ converges to a value close to $x_{\mathrm{sb}}^{\mathrm{r}}$, despite the theoretical condition $x_{\mathrm{sb}}(\infty) \neq x_{\mathrm{sb}}^{\mathrm{r}}$ as $K \rightarrow \infty$. Note that $K \rightarrow 0$ corresponds to disconnecting the regulator and the settler will overflow. 

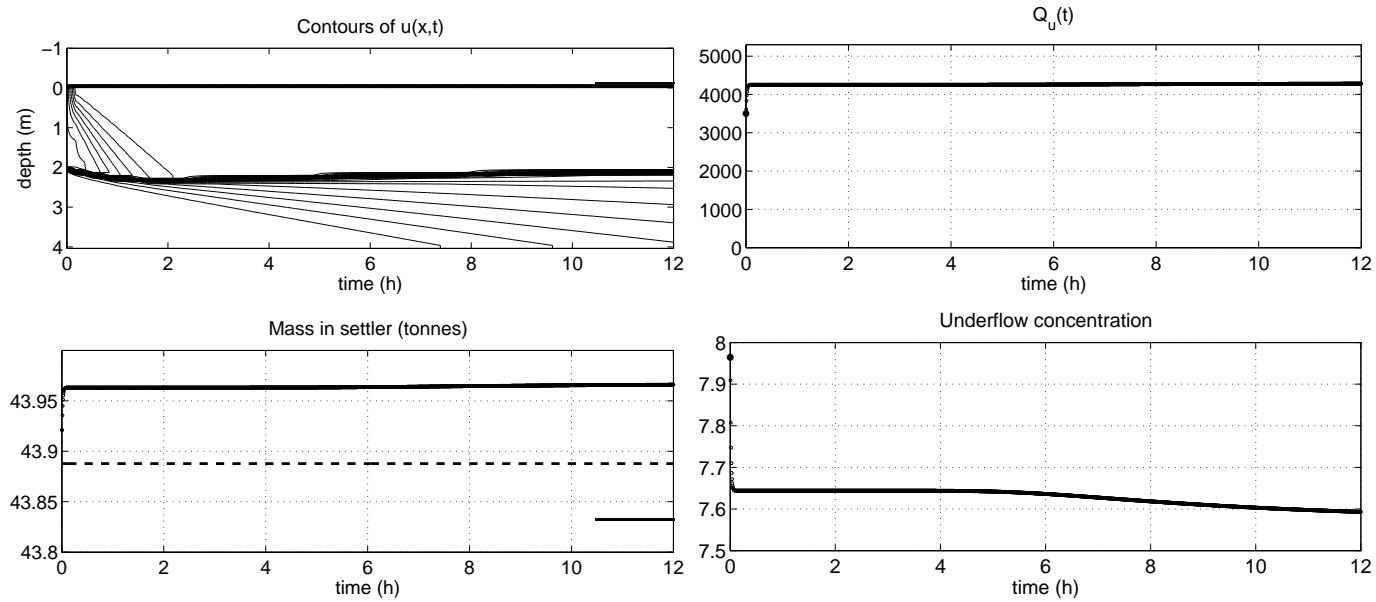

Figure 14. A simulation using the same data as in Figure 13, but with $K=10$ instead. The regulator implies that $m(t) \rightarrow 43.97$ tonnes and $x_{\mathrm{sb}}(t) \rightarrow 1.95 \mathrm{~m}$ as $t \rightarrow \infty$.
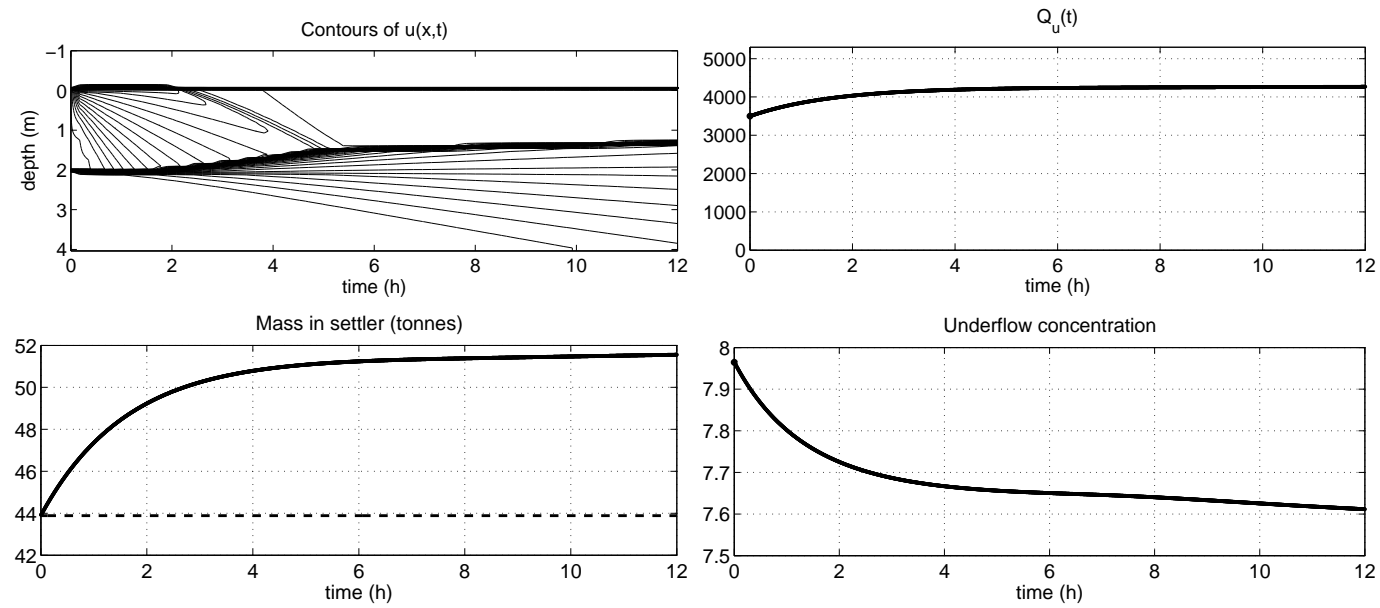

Figure 15. A simulation using the same data as in Figure 13, but with $K=0.1$ instead. The regulator implies that $m(t) \rightarrow 51.9$ tonnes and $x_{\mathrm{sb}}(t) \rightarrow 1.08 \mathrm{~m}$ as $t \rightarrow \infty$.

\section{A nonlinear regulator}

\subsection{THE REGULATOR}

To ensure that optimal operation is maintained, $Q_{\mathrm{u}}(t)$ has to be partly less than $\overline{\bar{Q}}_{\mathrm{u}}$ (by definition of optimal operation), partly not too small to avoid particles in the clarification zone. In other words, the proportional regulator (9) may saturate. Therefore, we introduce the following nonlinear regulator to satisfy the control objective COSBL1 or COSBL2, see Figure 16. We assume that the feed point satisfies (3) and that the mass is calculated continuously by (7).

Firstly, define $Q_{\mathrm{u}}^{\mathrm{r}}(t)$ by either (4) or (5). Secondly, for a given $x_{\mathrm{sb}}^{\mathrm{r}}$, set the reference mass $m^{\mathrm{r}}(t)$ according to (6). Thirdly, define

$$
Q_{\mathrm{u}}(t)=\min \left(Q_{\mathrm{u}}^{\max }, \max \left(Q_{\mathrm{u}}^{\min }(t), Q_{\mathrm{u}}^{\mathrm{r}}(t)+K\left(m(t)-m^{\mathrm{r}}(t)\right)\right)\right),
$$




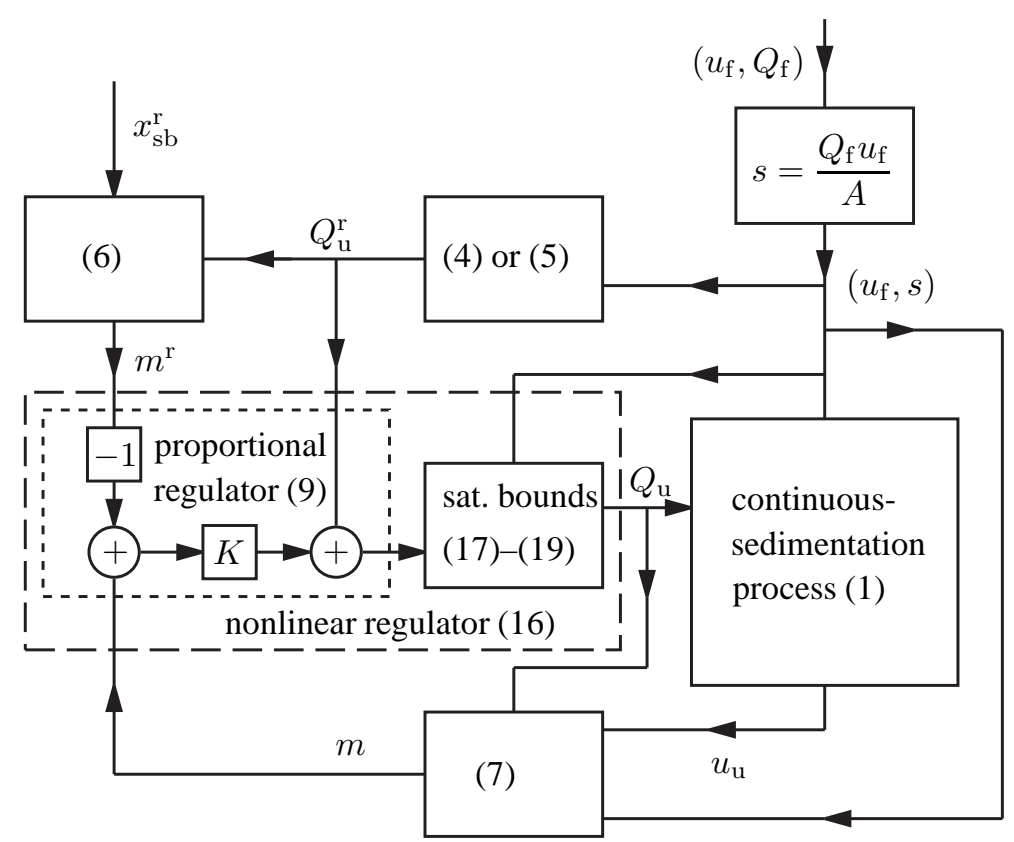

Figure 16. The closed-loop system of the clarification-thickening process with the regulator, feed forward and feedback loops.

where $Q_{\mathrm{u}}^{\max }$ and $Q_{\mathrm{u}}^{\min }(t)$ are saturating bounds, which are defined as follows and commented upon below. Firstly, set

$$
Q_{\mathrm{u}}^{\max }= \begin{cases}\overline{\bar{Q}}_{\mathrm{u}}, & \text { COSBL1 }, \\ Q_{\mathrm{u}}^{\max 1}, & \text { COSBL2 } .\end{cases}
$$

Secondly, let $Q_{\mathrm{u}}^{\min }(t)$ satisfy either

$$
Q_{\mathrm{u}}^{\min 1} \leq Q_{\mathrm{u}}^{\min }(t) \leq Q_{\mathrm{u}}^{\max } \quad \text { and } \quad\left(u_{\mathrm{f}}(t), s(t)\right) \in \mathcal{S}_{1}\left(Q_{\mathrm{u}}^{\min }(t)\right) \quad \text { (theoretically safe) }
$$

or

$$
Q_{\mathrm{u}}^{\min }(t)=\min \left(Q_{\mathrm{u}}^{\max }, \max \left(Q_{\mathrm{u}}^{\min 1}, L_{3}^{-1}\left(u_{\mathrm{f}}(t), s(t)\right)\right)\right) \quad \text { (less restrictive) }
$$

where $Q_{\mathrm{u}}^{\min 1}$ is a small positive number.

Considering COSBL1, $Q_{\mathrm{u}}^{\max }$ should not exceed $\overline{\bar{Q}}_{\mathrm{u}}$ by the definition of optimal operation ${ }^{1}$. For COSBL2 we set $Q_{\mathrm{u}}^{\max }=Q_{\mathrm{u}}^{\max 1}<\overline{\bar{Q}}_{\mathrm{u}}$ in accordance with Theorem 2.1 to meet the constraint $u_{\mathrm{u}}(t) \geq u_{\mathrm{u}}^{\min }$. In a plant there may be other reasons for defining a lower upper bound, for example, a limited pump capacity.

\footnotetext{
${ }^{1}$ In Definition 2.1 (optimal operation), we have required $Q_{\mathrm{u}}(t)<\overline{\bar{Q}}_{\mathrm{u}}$ instead of $Q_{\mathrm{u}}(t) \leq \overline{\bar{Q}}_{\mathrm{u}}$, cf. (17). The difference is subtle and of no practical importance. The only reason for the definition is that there exists no steadystate solution with a discontinuity in the thickening zone as $Q_{\mathrm{u}} \geq \bar{Q}_{\mathrm{u}}$, see [1]. During dynamic operation with a varying $Q_{\mathrm{u}}(t)$, the solution may satisfy all other requirements of Definition 2.1 despite $Q_{\mathrm{u}}(t) \geq \overline{\bar{Q}}_{\mathrm{u}}$ during a bounded time period.
} 
The reasons for the two alternative definitions, (18) and (19), of the lower bound $Q_{\mathrm{u}}^{\min }(t)$ are given in [4]. The theoretically safe (18) implies that optimal operation is not left. There are other less restrictive conditions for this, but these require more information, for example, the actual concentration distribution in the thickening zone. Hence, $Q_{\mathrm{u}}^{\mathrm{min}}$ should be set to the smallest possible value satisfying $\left(u_{\mathrm{f}}, s\right) \in \mathcal{S}_{1}\left(Q_{\mathrm{u}}^{\min }\right)$. In many cases $Q_{\mathrm{u}}^{\min }$ can be chosen such that $\left(u_{\mathrm{f}}, s\right)$ lies on the horizontal boundary between $\mathcal{S}_{1}$ and $\mathcal{S}_{2}$, which means that $s(t)=$ $f\left(u_{\mathrm{infl}}, Q_{\mathrm{u}}^{\min }(t)\right)$, see Figure 3. If the value of $s$ is so low that $\left(u_{\mathrm{f}}, s\right) \in \mathcal{S}_{1}\left(Q_{\mathrm{u}}=0\right)$, then we set $Q_{\mathrm{u}}^{\min }$ to a small positive value $Q_{\mathrm{u}}^{\min 1}$. Recall that we have assumed that $Q_{\mathrm{u}}(t)>0$, since $u_{\mathrm{u}}(t)$ is undefined as $Q_{\mathrm{u}}(t)=0$. In a plant there may be other reasons for choosing $Q_{\mathrm{u}}^{\min }$ not too close to zero.

A high value on $s$ implies a high value on $Q_{\mathrm{u}}^{\min }$, which may imply a fast declining SBL and a low underflow concentration (cf. Theorem 2.1). Then the less restrictive condition that $\left(u_{\mathrm{f}}, s\right) \in \mathcal{S}$ may be advantageous. This yields namely the lower value $Q_{\mathrm{u}}^{\min }(t)=L_{3}^{-1}\left(u_{\mathrm{f}}(t), s(t)\right)$ (if this is positive; otherwise set $Q_{\mathrm{u}}^{\min }(t)=Q_{\mathrm{u}}^{\min 1}>0$ ). The drawback is that there is an exceptional case, in which there are some particles in the lower part of the clarification zone during a limited time period. This is believed to occur only rarely and the advantage of this lower value of $Q_{\mathrm{u}}^{\min }$ is believed to be more important in the applications. Therefore, we prefer (19) in the examples below.

Since either of the two minimum bounds described above may be greater than $Q_{\mathrm{u}}^{\max 1}$ defined in Theorem 2.1 to satisfy COSBL2, the requirement $Q_{\mathrm{u}}^{\min }(t) \leq Q_{\mathrm{u}}^{\max }$ is included in both (18) and (19). Hence, (16) implies that $Q_{\mathrm{u}}^{\min }(t) \leq Q_{\mathrm{u}}(t) \leq Q_{\mathrm{u}}^{\max }$ holds.

Example. Assume that the initial data and step input are the same as in the example in Section 4.2, except for the location of the SBL, which is now close to the bottom. A simulation with the regulator (16) is shown in Figure 17. During the first 9 hours the mass is less than its reference value and the control variable takes its lowest possible value $Q_{\mathrm{u}}(t)=$ $Q_{\mathrm{u}}^{\min }=3876 \mathrm{~m}^{3} / \mathrm{h}$. Any lower value of $Q_{\mathrm{u}}(t)$ would imply particles in the clarification zone. The advantage of controlling the mass (instead of controlling the SBL directly) is here illustrated clearly. After 9 hours the control variable converges quickly to its final value $Q_{\mathrm{u}}^{\mathrm{r}}=$ $L_{1}^{-1}(3,11.5)=4298 \mathrm{~m}^{3} / \mathrm{h}$. During 3 hours further the SBL rises and then reaches its reference level.

\subsection{SOME PROPERTIES OF THE NONLINEAR REGULATOR}

By numerical simulations we shall illustrate some properties of the regulator (16) given the periodic indata in [4, Examples 1 and 2], see Figures 18 and 19. In particular, the influence of the saturating bounds is demonstrated. Let $x_{\mathrm{sb}}^{\mathrm{r}}=2 \mathrm{~m}$ and $Q_{\mathrm{u}}^{\min }(t)$ be defined by (19), with $Q_{\mathrm{u}}^{\min 1}=1 \mathrm{~m}^{3} / \mathrm{h}$, in the following cases:
A. $Q_{\mathrm{u}}^{\mathrm{r}}(t)$ is defined by (4), $K=1$, and $Q_{\mathrm{u}}^{\max }=\overline{\bar{Q}}_{\mathrm{u}}$ to satisfy COSBL1,
B. $Q_{\mathrm{u}}^{\mathrm{r}}(t)$ is defined by (5) with $T=4, K=1$, and $Q_{\mathrm{u}}^{\max }=\overline{\bar{Q}}_{\mathrm{u}}$ to satisfy COSBL1,
C. $Q_{\mathrm{u}}^{\mathrm{r}}(t)$ is defined by (5) with $T=4, K=0.1$ and $Q_{\mathrm{u}}^{\max }=\overline{\bar{Q}}_{\mathrm{u}}$ to satisfy COSBL1,
D. $Q_{\mathrm{u}}^{\mathrm{r}}(t)$ is defined by (4), $K=1$ and $Q_{\mathrm{u}}^{\max }=Q_{\mathrm{u}}^{\max 1}$ to satisfy COSBL2.

Example 1, case A. In Figure 20, a simulation shows how the regulator influences the periodic input in order to satisfy COSBL1. Right after each jump in the feed point, $Q_{\mathrm{u}}(t)$ makes 

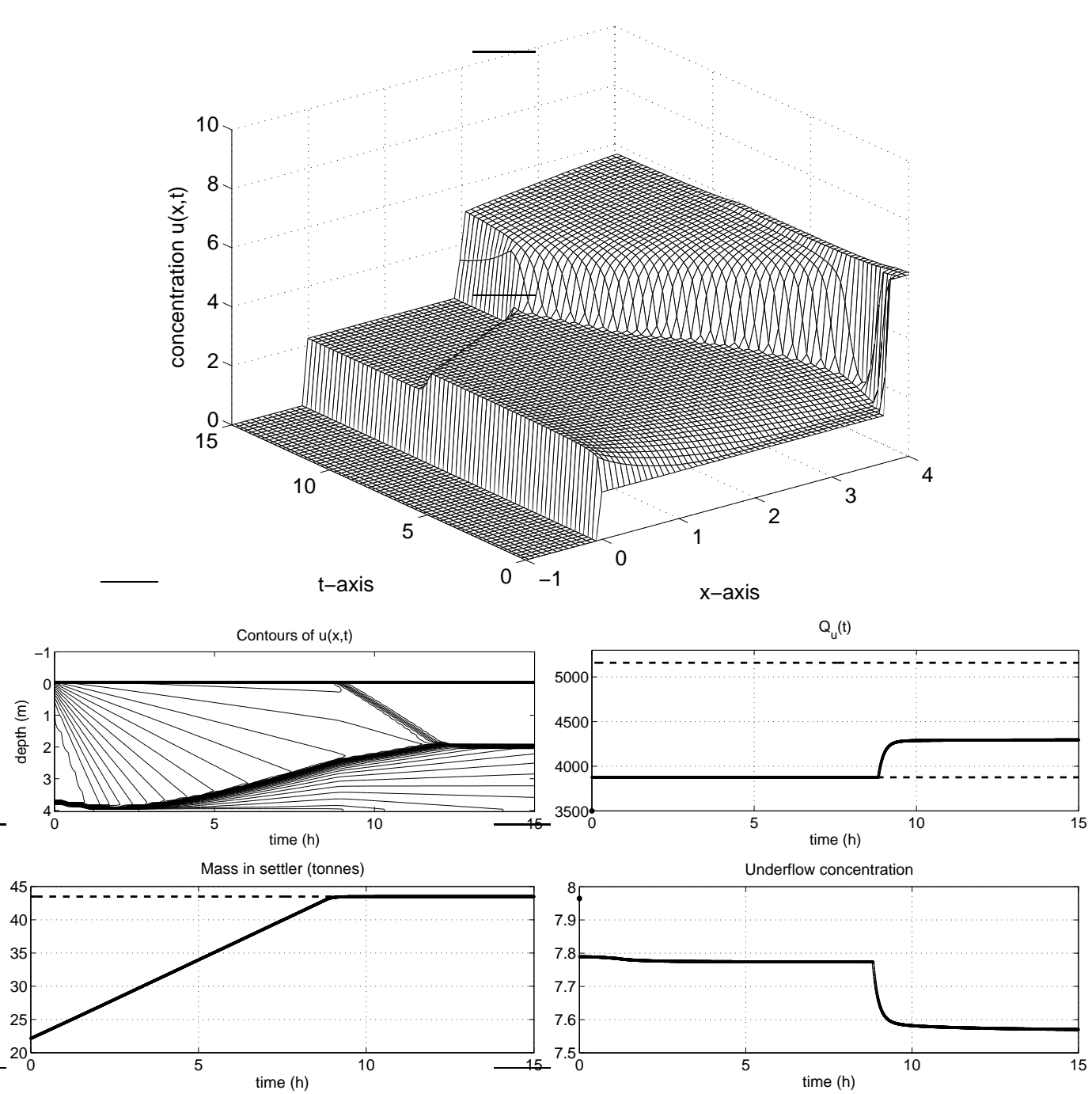

Figure 17. A 15-hour-simulation using the same initial data and step input as in Figure 11, except for the initial SBL; $x_{\mathrm{sb} 0}=3.7 \mathrm{~m}$. At $t=0$ there is a step change from $\left(u_{\mathrm{f} 0}, s_{0}\right)=(3,9.86)$ to $\left(u_{\mathrm{f}}, s\right)=(3,11.5) \in \mathcal{O}\left(Q_{\mathrm{u} 0}\right) \cap \Lambda_{2}$. The regulator (16) is connected with $K=1, x_{\mathrm{sb}}^{\mathrm{r}}=2$, $Q_{\mathrm{u}}^{\mathrm{r}}=L_{1}^{-1}(3,11.5)=4298, Q_{\mathrm{u}}^{\max }=\overline{\bar{Q}}_{\mathrm{u}}=5159$ and $Q_{\mathrm{u}}^{\min }=L_{3}^{-1}(3,11.5)=3876$. The two latter values are shown by dashed lines. The reference mass $m^{\mathrm{r}}(t)=43.5$ is shown by the dashed line. COSBL1 is satisfied and the SBL is adjusted to the desired level after $12 \mathrm{~h}$.

a large jump and stays, during a short time, at the saturating value $Q_{\mathrm{u}}^{\min }(t)=Q_{\mathrm{u}}^{\min 1}=1$ at $t=0,4,8, \ldots$, and $Q_{\mathrm{u}}^{\max }$ at $t=2,6,10, \ldots$. These large jumps are caused by the jumps in the reference value $Q_{\mathrm{u}}^{\mathrm{r}}(t)$ by (4). Note that a jump in $Q_{\mathrm{u}}^{\mathrm{r}}(t)$ also implies a jump in $m^{\mathrm{r}}(t)$; see (6).

Example 1, case B. When defining $Q_{\mathrm{u}}^{\mathrm{r}}(t)$ by (5) with the time average taken over a period ( $T=4 \mathrm{~h}$ ), it will be constant and equal to the initial value $Q_{\mathrm{u} 0}$. This corresponds to the initial feed point, which is the mean value of the periodic input, see Figure 18 (upper left). Thus, $Q_{\mathrm{u}}^{\mathrm{r}}=Q_{\mathrm{u} 0}=L_{1}^{-1}(2.5,7.5)=2488$ and $m^{\mathrm{r}}(t)=45.6$. Then $Q_{\mathrm{u}}(t)$ depends continuously on time unless it has to jump because of the saturating bounds. This is demonstrated in Figure 21, where small jumps in $Q_{\mathrm{u}}(t)$ can be seen at $t=2,6,10, \ldots$. At each of these time points the 

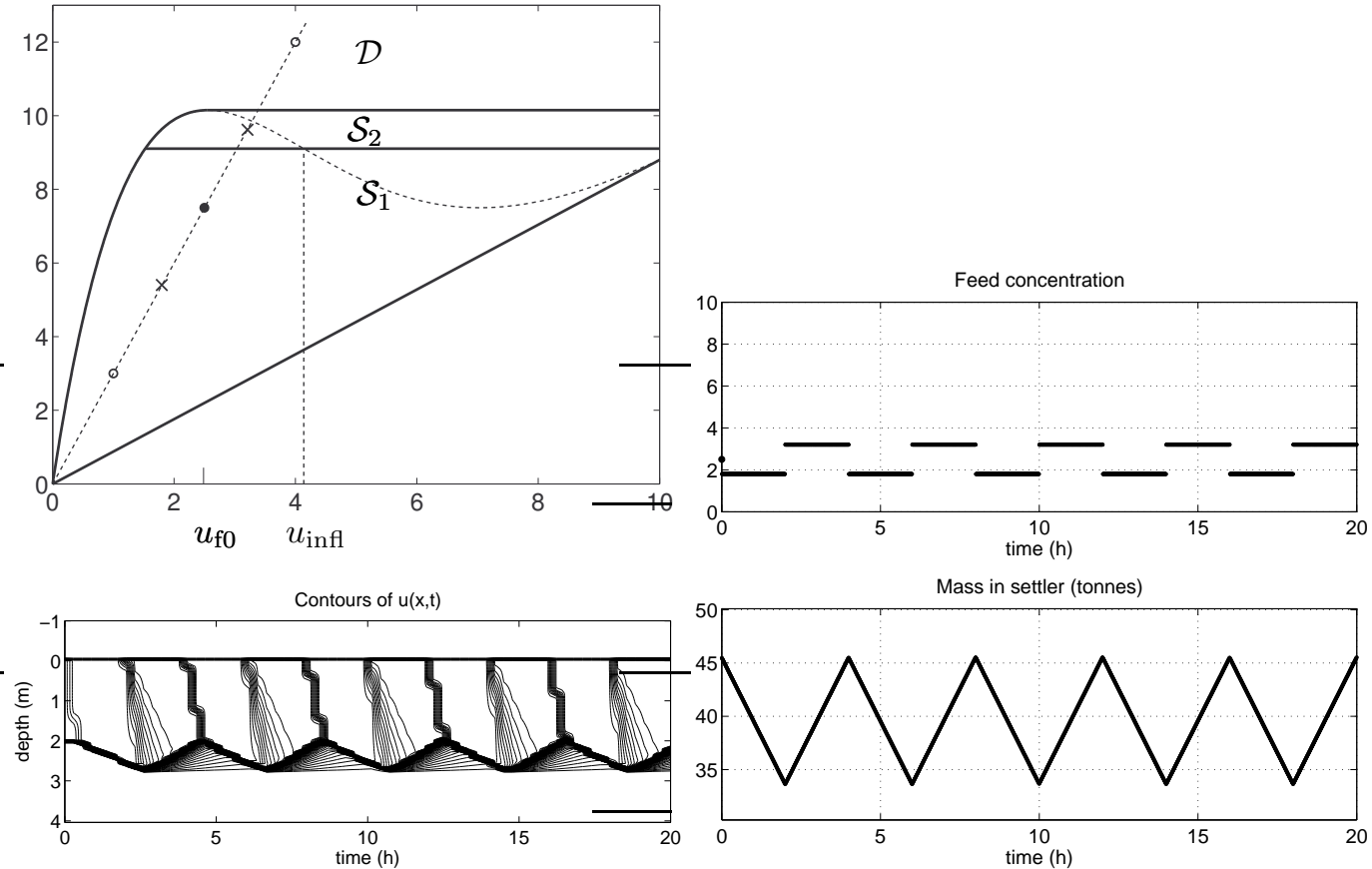

Figure 18. Upper left: Operating chart as $Q_{\mathrm{u}}=Q_{\mathrm{u} 0}=2488 \mathrm{~m}^{3} / \mathrm{h}$. Located on the dashed feed line $y=\frac{Q_{\mathrm{f}}}{A} u$ are the feed points of Example 1, the crosses, and Example 2, circles. The filled dot is the initial feed point $\left(u_{\mathrm{f} 0}, s_{0}\right)=(2.5,7.5)$. Upper right and lower row: Example 1. A numerical simulation (without a regulator) when the feed concentration is piecewise constant and periodic with the alternating values 1.8 and $3.2 \mathrm{~kg} / \mathrm{m}^{3}$. $Q_{\mathrm{f}}(t)=8482 \mathrm{~m}^{3} / \mathrm{h}, Q_{\mathrm{u}}(t)=Q_{\mathrm{u} 0}=L_{1}^{-1}\left(u_{\mathrm{f} 0}, s_{0}\right)=2488 \mathrm{~m}^{3} / \mathrm{h}, u_{\mathrm{u}}(t)=u_{\mathrm{u} 0}=8.52 \mathrm{~kg} / \mathrm{m}^{3}$ and $u_{\mathrm{e}}(t)=0$.

high value of the feed flux $s$ implies a jump from $Q_{\mathrm{u}}(2-)=1688\left(\right.$ when $Q_{\mathrm{u}}^{\min }(2-)=1$ ) up to $Q_{\mathrm{u}}(2)=Q_{\mathrm{u}}^{\min }(2)=1860$. The amplitudes of $Q_{\mathrm{u}}(t), m(t), u_{\mathrm{u}}(t)$ and $x_{\mathrm{sb}}(t)$ are now smaller
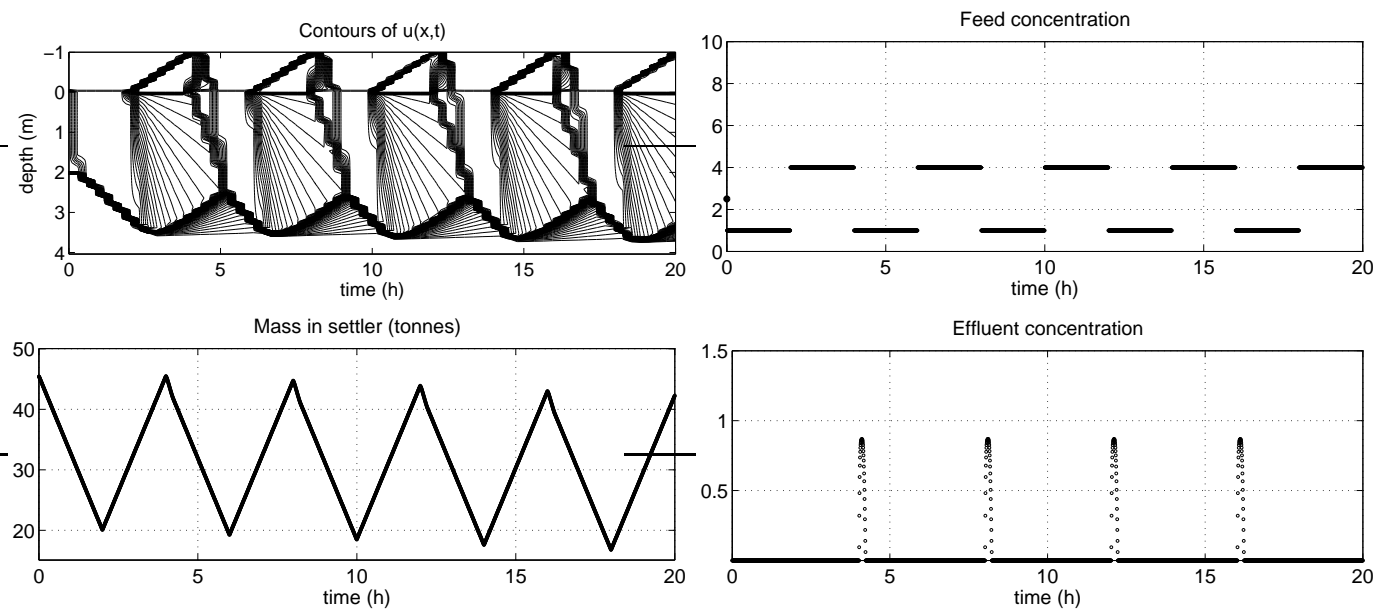

Figure 19. Example 2. A simulation (without a regulator) where the alternating values of the periodic feed concentration are 1 and $4 \mathrm{~kg} / \mathrm{m}^{3}$. This larger amplitude than in Example 1 implies overflow and a slightly declining SBL and mass, on an average. $Q_{\mathrm{f}}(t)=8482,\left(u_{\mathrm{f} 0}, s_{0}\right)=(2.5,7.5), Q_{\mathrm{u}}(t)=Q_{\mathrm{u} 0}=L_{1}^{-1}\left(u_{\mathrm{f} 0}, s_{0}\right)=2488$ and $u_{\mathrm{u}}(t)=u_{\mathrm{u} 0}=8.52$. 

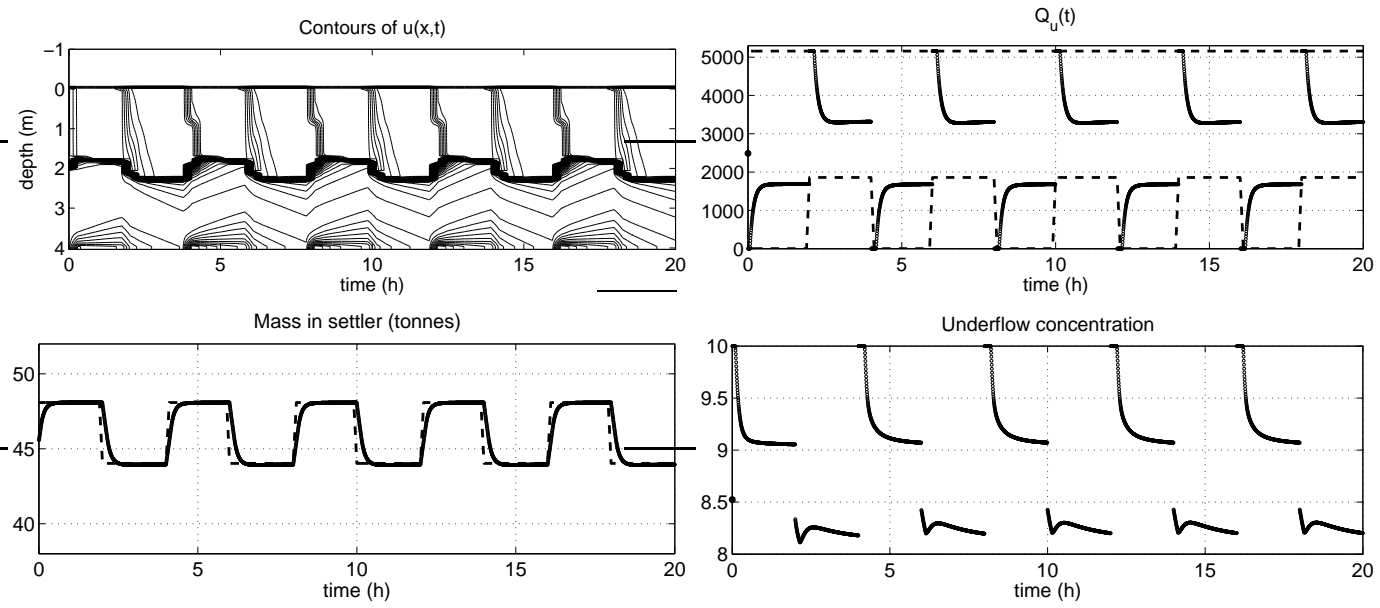

Figure 20. Example 1, case A. A simulation using the same initial data and alternating feed concentration as in Figure 18. The regulator (16) is applied with $K=1, Q_{\mathrm{u}}^{\max }=\overline{\bar{Q}}_{\mathrm{u}}=5159$, and the alternating values of $Q_{\mathrm{u}}^{\mathrm{r}}(t)$ are $L_{1}^{-1}(1.8,5.4)=1688$ and $L_{1}^{-1}(3.2,9.6)=3382$, respectively. The alternating values of $Q_{\mathrm{u}}^{\min }(t)$ are $Q_{\mathrm{u}}^{\min 1}=1$ and $L_{3}^{-1}(3.2,9.6)=1860$, respectively, see the dashed lines. COSBL1 is satisfied.
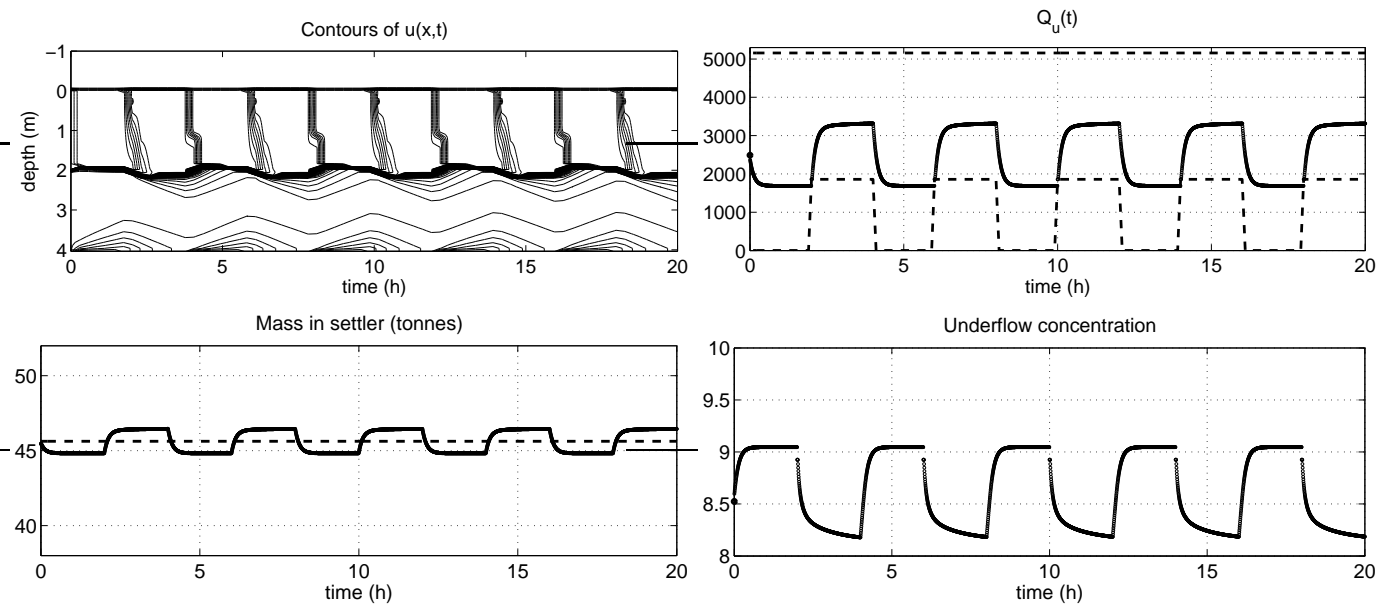

Figure 21. Example 1, case B. A simulation using the same initial data and alternating feed concentration as in Figure 20. The regulator (16) is applied with $K=1$ and the constant value $Q_{\mathrm{u}}^{\mathrm{r}}=Q_{\mathrm{u} 0}=2488$. The alternating values of $Q_{\mathrm{u}}^{\min }(t)$ are $Q_{\mathrm{u}}^{\min 1}=1$ and 1860, see the dashed lines. COSBL1 is satisfied.

than in Figure 20. Note that the constant $Q_{\mathrm{u}}^{\mathrm{r}}$ implies the following phenomenon. Since the jump down from $s_{0}=7.5$ to $s(t)=5.4$ (for $0<t<2$ ) implies that $m(t)<m^{\mathrm{r}}$, the regulator decreases $Q_{\mathrm{u}}(t)$ from $Q_{\mathrm{u} 0}=2488$. After a short while it converges to $L_{1}^{-1}(1.8,5.4)=1688$, which is precisely the value that corresponds to a steady-state solution in optimal operation (with equal mass flux in and out), cf. Theorem 4.1. The analogous behaviour occurs during the periods when the feed flux takes the high value $s(t)=9.6\left(2<t<4\right.$, etc.). Then $Q_{\mathrm{u}}(t)$ increases and converges to $L_{1}^{-1}(3.2,9.6)=3382$. In accordance with (13), the mass also converges to a constant value, different from $m^{\mathrm{r}}(t)$, after each jump.

Example 1, case $C$. A more sluggish behaviour of $Q_{\mathrm{u}}(t)$ can be obtained by decreasing the regulator gain $K$, see Figure 22. In comparison to Figure 21 (where $K=1$ ), the value $K=0.1$ 

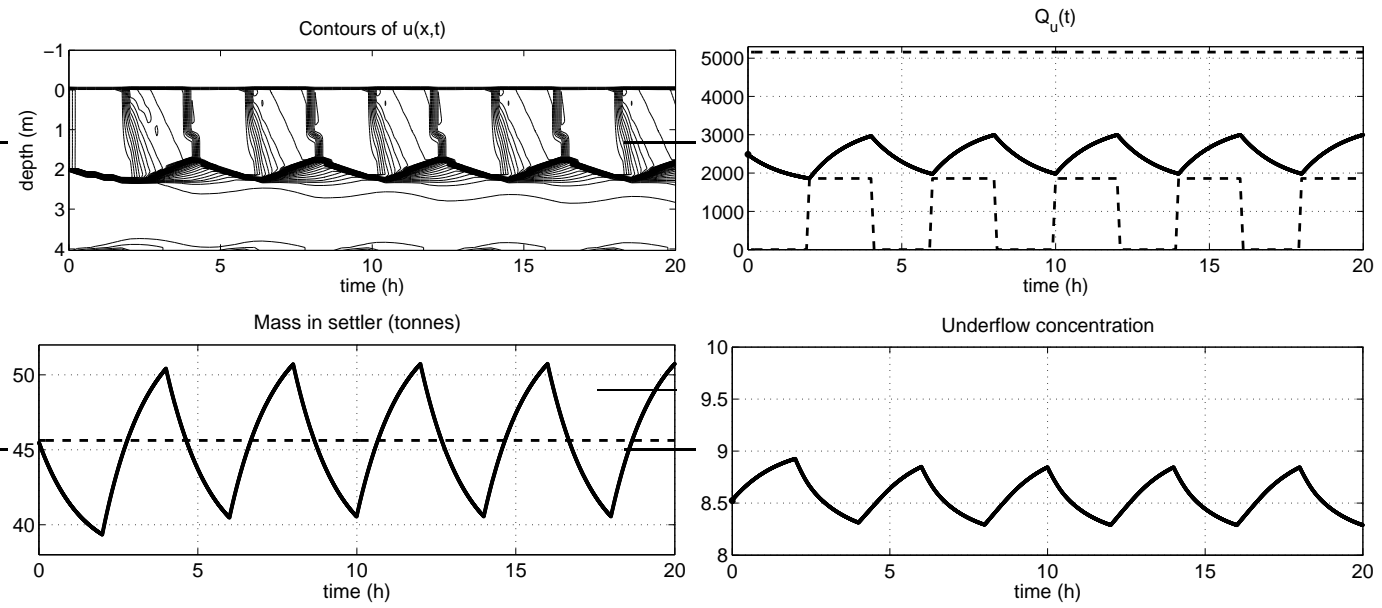

Figure 22. Example 1, case C. A simulation using the same conditions as in Figure 21, but with $K=0.1$ instead. COSBL1 is satisfied.

implies that $Q_{\mathrm{u}}(t)$ and $u_{\mathrm{u}}(t)$ are continuous and show a more sluggish behaviour to the price of increased amplitudes in $m(t)$ and $x_{\mathrm{sb}}(t)$.

Example 1, case D. Consider now the control objective COSBL2. The initial underflow concentration is $u_{\mathrm{u} 0}=8.52$ and belongs to the optimal-operation steady-state solution for $\left(u_{\mathrm{f} 0}, s_{0}\right)$, which is the mean value of the periodically varying feed point. Setting $u_{\mathrm{u}}^{\min }=8.5$ would thus be a hard constraint to fulfil. This corresponds to the rather low maximal bound $Q_{\mathrm{u}}^{\max 1}=2528$, cf. Figure 4. Nevertheless, the simulation in Figure 23 shows that COSBL2 is satisfied.
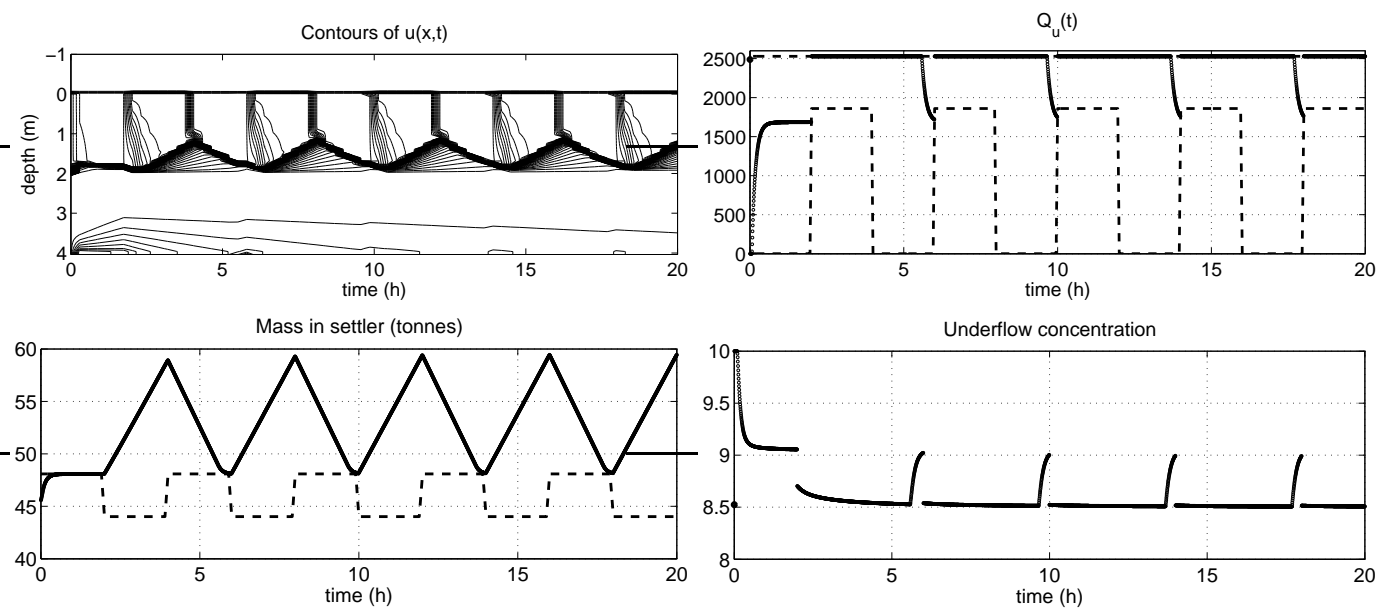

Figure 23. Example 1, case D. A simulation using the same conditions as in Figure 20, but with the additional requirement $u_{\mathrm{u}}(t) \geq u_{\mathrm{u}}^{\min }=8.5$, which is implied by $Q_{\mathrm{u}}(t) \leq Q_{\mathrm{u}}^{\max 1}=2528$. COSBL2 is satisfied.

Example 2, case A. Despite the large jumps in the periodic input (see the circles in Figure 18) the regulator can handle the situation when $K=1$, see Figure 24. 

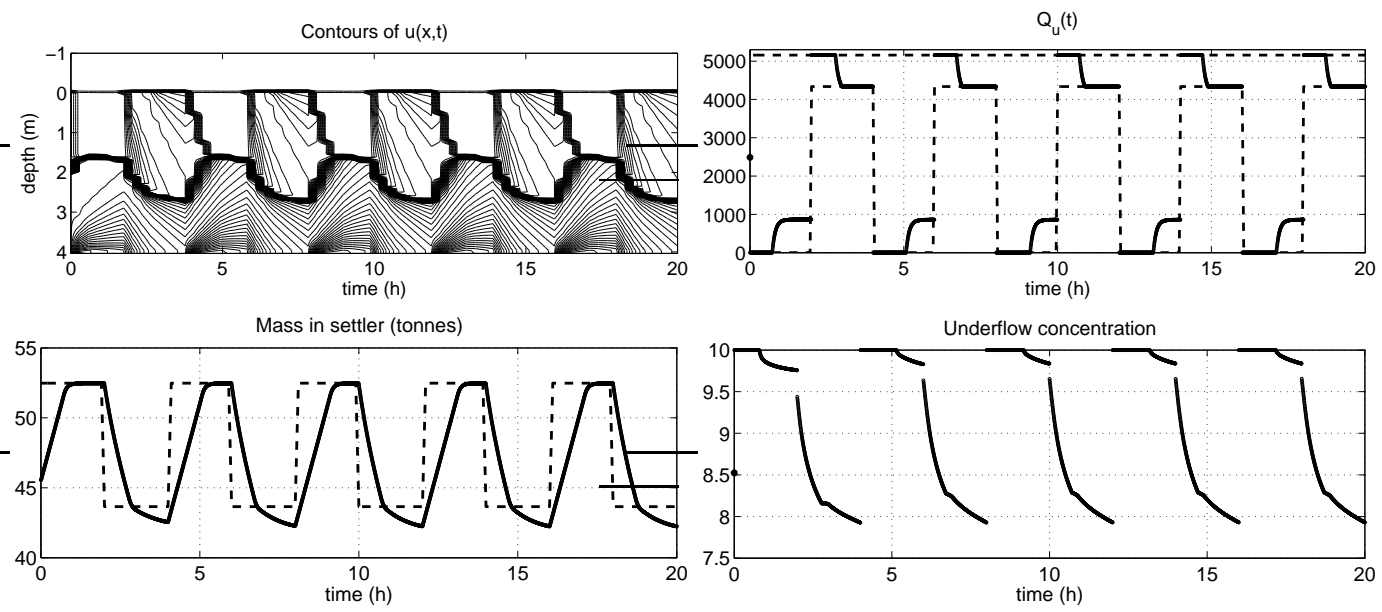

Figure 24. Example 2, case A. A simulation using the same initial data and alternating $u_{\mathrm{f}}(t)$ as in Figure 19. The regulator (16) is applied with $K=1$ and the alternating values of $Q_{\mathrm{u}}^{\min }(t)$ are $Q_{\mathrm{u}}^{\min 1}=1$ and $L_{3}^{-1}(4,12)=4335$. Both these two latter values are assumed by the regulator, as well as the upper bound $Q_{\mathrm{u}}^{\max }(t)=\overline{\bar{Q}}_{\mathrm{u}}=5159$. Although the regulator saturates about half the time COSBL1 is satisfied.

Example 2, case B-C. Setting $Q_{\mathrm{u}}^{\mathrm{r}}(t)=Q_{\mathrm{u} 0}=2488$, which is the value corresponding to a steady-state solution in optimal operation for the mean value of the two input feed points, we get the solution shown in Figure 25 for $K=1$ and Figure 26 for $K=0.1$.
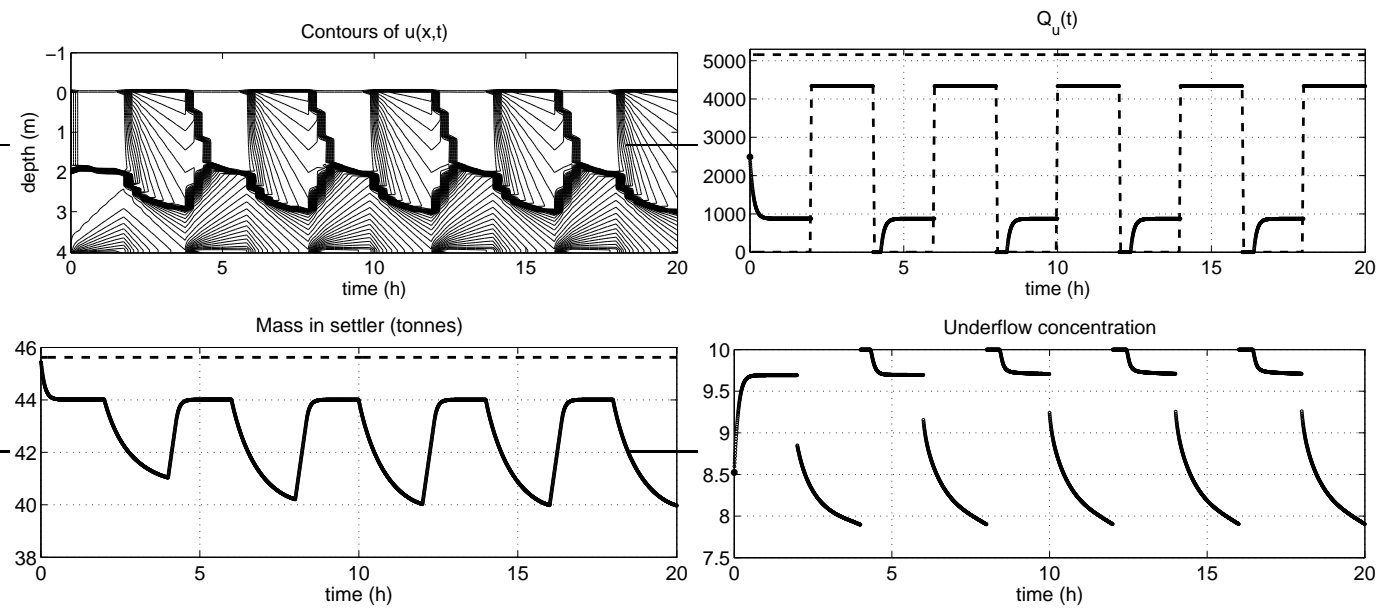

Figure 25. Example 2, case B. A simulation using the same conditions as in Figure 24, but with the constant mean value $Q_{\mathrm{u}}^{\mathrm{r}}(t)=Q_{\mathrm{u} 0}=2488$ instead. During the whole intervals of high load $(2<t<4,6<t<8$, etc.) the regulator saturates to $Q_{\mathrm{u}}(t)=Q_{\mathrm{u}}^{\min }(t)=L_{3}^{-1}(4,12)=4335$. During the other intervals, however, convergence to $Q_{\mathrm{u}}(t)=L_{1}^{-1}(1,3)=875$ occurs. Although the average mass over a period decreases initially, longer simulation times show that it converges to a constant value and that COSBL1 is satisfied.

Example 2, case D. Suppose that COSBL2 is required with $u_{\mathrm{u}}^{\min }=8.5$ as in Example 1D. The constraint $Q_{\mathrm{u}}(t) \leq Q_{\mathrm{u}}^{\max 1}=2528$ implies that there will be particles in the clarification zone during the high load intervals, since the minimum bound $L_{3}^{-1}(4,12)=4335>Q_{\mathrm{u}}^{\max 1}$. A simulation is shown in Figure 27. There will be an overflow with effluent concentrations similar to Figure 19, where $Q_{\mathrm{u}}(t)=2488$ (for $t>0$ ), which is close to $Q_{\mathrm{u}}^{\max 1}=2528$. In 

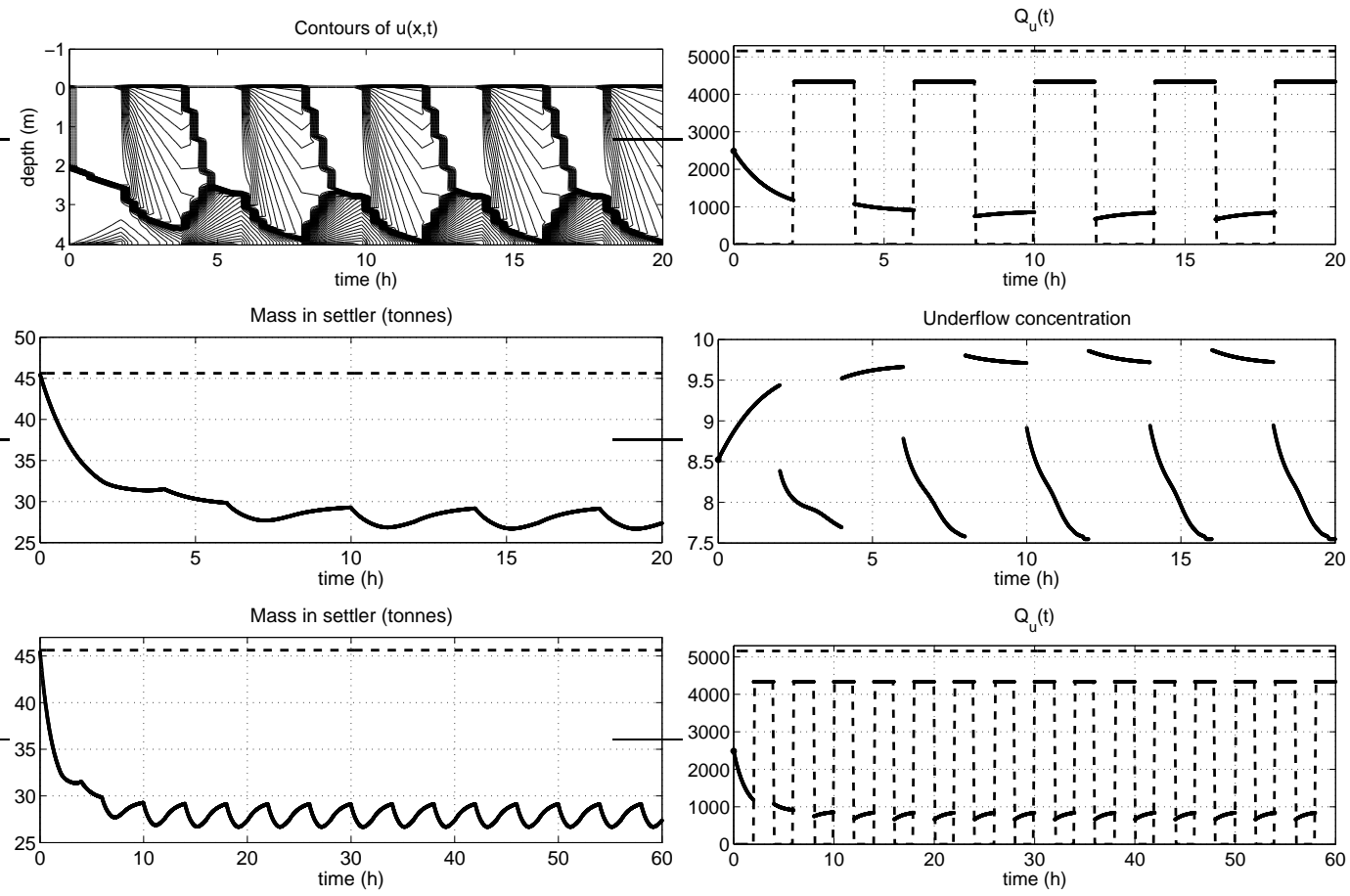

Figure 26. Example 2, case C. A simulation using the same conditions as in Figure 25, but with $K=0.1$ instead. The behaviour of $Q_{\mathrm{u}}(t)$ is now more sluggish and the average mass decreases initially, however, simulation longer (see the graphs in the third row) reveals that it converges to a value such that the SBL touches the bottom, although the average SBL lies within the thickening zone.

Figure 19, there is no regulator connected and the underflow concentration is constant $u_{\mathrm{u}}(t)=$ 8.52. However, the mass decreases and the SBL reaches the bottom. The major improvement with the regulator connected is that the SBL is maintained within the thickening zone, which can be confirmed by longer simulation times.

\section{Concluding discussions}

The main result in this paper is the nonlinear regulator (16), see the closed-loop system in Figure 16. It consists of a proportional regulator and saturating bounds. The underlying ideas and results originate from the preceding series of papers [1-4].

The control objectives we have focused on are to maintain optimal operation and keep the SBL at a prescribed level, with and without a constraint on the underflow concentration, see Section 3.1.

A necessary condition for maintaining optimal operation during long time of dynamic operation is that the feed point satistfies (3): $\left(u_{\mathrm{f}}(t), s(t)\right) \in P_{1} \cup \Lambda_{2} \cup \Lambda_{3 \mathrm{a}}$. We have in [4] motivated why it is reasonable to assume this. If it is not satisfied, the feed concentration is either too high, or the settler is underdimensioned. Then $Q_{\mathrm{u}}$ has to be increased sufficiently (above $\overline{\bar{Q}}_{\mathrm{u}}$ ) to prevent overflow; see [3], where the control of step responses cover all cases.

The responses of the process to small disturbances from optimal operation can be found in Section 3.2. They constitute a fundamental property of the process, which is well known among all operators of clarifier-thickeners: an increase in the control variable will result in 

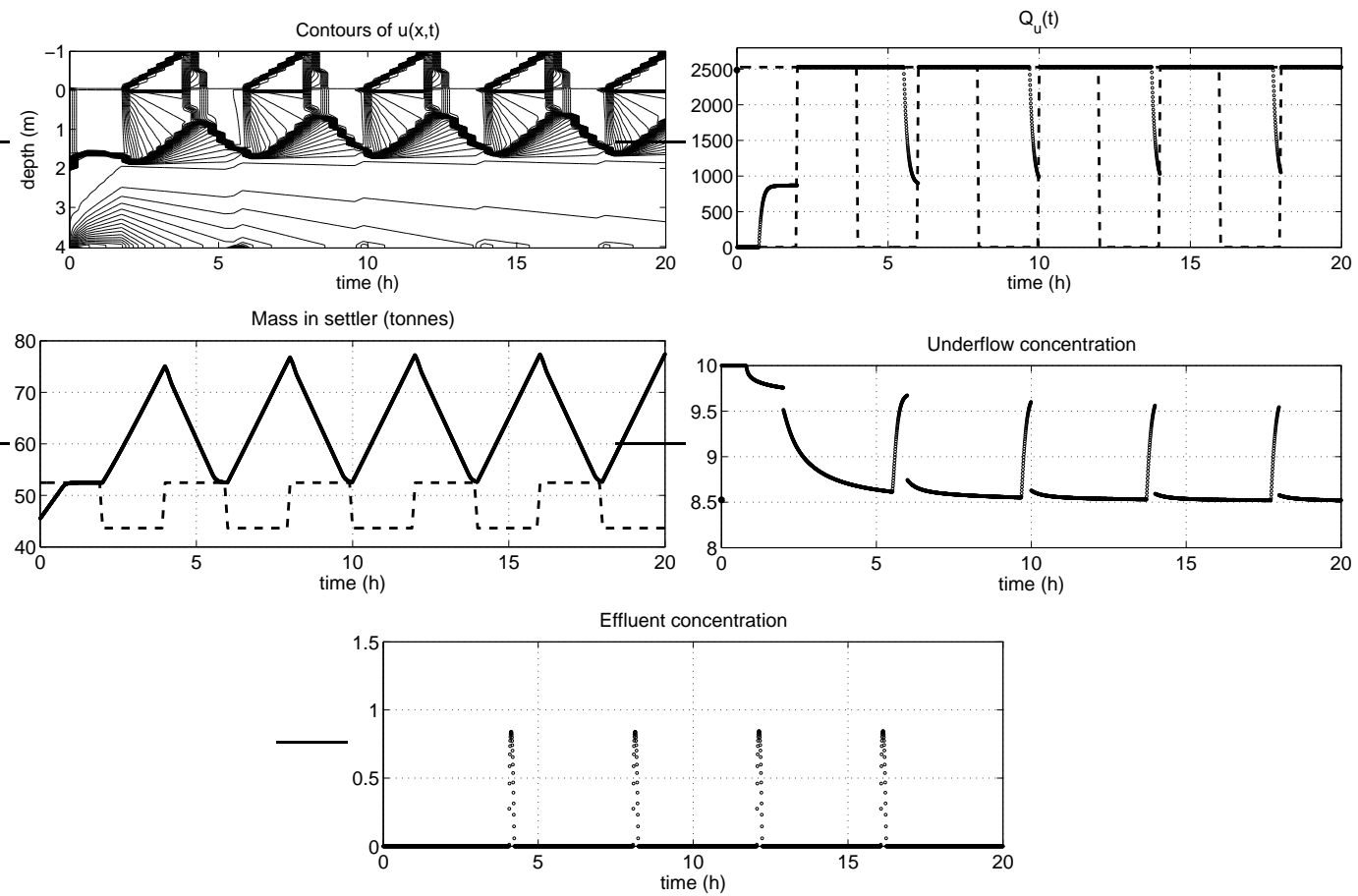

Figure 27. Example 2, case D. A simulation using the same conditions as in Figure 24, but with the additional requirement $u_{\mathrm{u}}(t) \geq u_{\mathrm{u}}^{\min }=8.5$, which is implied by $Q_{\mathrm{u}}(t) \leq Q_{\mathrm{u}}^{\max 1}=2528$. This requirement means that the interval of the saturating bound is reduced to the single value 2528 during the intervals of high load. The settler overflows periodically at the end of these intervals.

a decrease in mass and a declining SBL, and vice versa. Note that this conclusion cannot be drawn from the explicit formula (8), since $u_{\mathrm{u}}(t)$ increases as $Q_{\mathrm{u}}(t)$ decreases, and vice versa. This property, together with the steady-state relation between the mass, the SBL and the control variable in Section 3.3, yields the first part of a control strategy, see Section 3.4. This part is realized by means of the proportional regulator (9), which controls the mass in the settler. The key idea is the following. Under dynamic conditions when the settler is in optimal operation, the solution is approximately like one in optimal operation in steady state. For the latter solution there is a known relation (2) between the mass, the SBL and the control variable. Hence, by controlling the settler such that optimal operation is maintained, the SBL can be controlled indirectly via the mass. The mass in the settler can be computed since we assume that the inlet and outlet concentrations and volume flows can be measured. In this way, the SBL can be controlled without measuring it. Furthermore, controlling the mass may be more advantageous, since the SBL may vary during a transient despite the mass is constant.

A favourable property of the relationship (2) between the mass, SBL and control variable is the following. For a constant control variable, (2) is an affine relationship between the mass and the SBL. When the reference value of the SBL is chosen to be in the middle of the thickening zone, (2) is almost constant as a function of the control variable, see Figure 9.

All numerical simulations performed by the author and found in the literature converge to steady-state solutions when the feed inputs and the control variable are held constant. The same seems to be true when the regulator (9) is connected and we conjecture that this is true. 
Without the regulator, the way of controlling the process is by adjusting $Q_{\mathrm{u}}(t)$ manually, see [3,4]. With the regulator (9), only the reference value $x_{\mathrm{sb}}^{\mathrm{r}}$ needs to be set. The reference value $Q_{\mathrm{u}}^{\mathrm{r}}(t)$ can be defined automatically by (4) or (5), and its value is not that crucial, since the process is much more stable. Situations when step responses would cause either overflow of particles or an underloaded settler with a low underflow concentration, are avoided by connecting the regulator. For a constant feed point, and no regulator connected, optimal operation is inevitably left after a finite time when $Q_{\mathrm{u}} \neq L_{1}^{-1}\left(u_{\mathrm{f}}, s\right)$ (see [2]). A steady-state solution in optimal operation is unstable in this sense, with the present hyperbolic model.

When the regulator is connected, Theorem 4.1 yields that a stationary solution in optimal operation becomes stable in the following sense. For a constant reference value, $Q_{\mathrm{u}}^{\mathrm{r}}=$ $L_{1}^{-1}\left(u_{\mathrm{f} 0}, s_{0}\right)$, and a step to $\left(u_{\mathrm{f}}, s\right)$, optimal operation is maintained for a whole range of values of $L_{1}^{-1}\left(u_{\mathrm{f}}, s\right)$ of the control parameter. The location of the limit SBL is given by the explicit formula (15), which is generally different from the reference value $x_{\mathrm{sb}}^{\mathrm{r}}$. As $Q_{\mathrm{u}}^{\mathrm{r}}$ is set to the value $L_{1}^{-1}\left(u_{\mathrm{f}}, s\right)$, see $(4)$, then the $\mathrm{SBL}$ converges to the desired $x_{\mathrm{sb}}^{\mathrm{r}}$. These properties are demonstrated by the simulations in Section 4.2.

In the examples in Section 4.2, the influence of the regulator gain $K>0$ is also demonstrated. When (4) is used to define $Q_{\mathrm{u}}^{\mathrm{r}}(t)$, then the transient behaviour is not very sensitive to $K$, unless it is not too small ( $K=0$ means that the regulator is disconnected). A too high value of $K$ implies, however, problems since $Q_{\mathrm{u}}$ in (9) then is substantially different from $Q_{\mathrm{u}}^{\mathrm{r}}$ even for small differences $m-m^{\mathrm{r}}$. If the latter difference also changes sign, there is a problem of a rapidly fluctuating $Q_{\mathrm{u}}$, which may not be desirable. When $Q_{\mathrm{u}}^{\mathrm{r}}(t)$ is defined by (5), then the value of $K$ influences the transient behaviour more as well as the limit SBL (given by (15)) in case the feed point is constant after a certain time point.

Consider the saturating bounds of the nonlinear regulator (16). The upper saturation bound $Q_{\mathrm{u}}^{\max 1}$ in (17) is defined in Theorem 2.1 and implies that the underflow concentration is bounded below, which is a constraint of a control objective in Section 3.1. Depending on the actual application there may be other upper bounds to take into account, for example, a limited pump capacity.

One obvious lower bound is that $Q_{\mathrm{u}}(t)$ cannot be negative, hence the constant $Q_{\mathrm{u}}^{\min 1}$ in (18) and (19). The reason for the lower bound $Q_{\mathrm{u}}^{\min }(t)$, defined by (18) or (19), is to avoid that optimal operation is left, see [4]. For feed concentrations which are not too high (above $u_{\mathrm{M}}$ ), this is equivalent to avoiding an upflow of particles in the clarification zone. We recommend the condition (19), since it implies a wider range for the regulator to work within. The drawback is that in an exceptional case, which is believed to occur only rarely, there may be some particles in the lower part of the clarification zone during a limited time period. In the simulations, we have used (19) without any problems.

The simulation in Figure 17 illustrates the advantage of controlling the mass instead of controlling the SBL directly. After 9 hours in that simulation, the mass in the settler has reached its reference value and the control variable converges quickly to its final value. During 3 hours further the SBL rises and then reaches its reference level. Hence, once the mass has reached its reference value, the transient solution in the thickening zone will automatically yield the desired SBL.

The performance of the nonlinear regulator (16) is further illustrated by several numerical simulations in Section 5.2. In particular, the influence of the saturating bounds are investigated. These are shown by dashed lines in the graphs of $Q_{\mathrm{u}}(t)$. Note that these bounds depend on time via the movement of the feed point. The two different choices of reference value of $Q_{\mathrm{u}}^{\mathrm{r}}(t)$, defined by (4) and (5), as well as different values on the regulator gain $K$, are investigated. 
For the moderate amplitude of the periodic feed point in Example 1 (Figure 18), the control objective COSBL1 is satisfied in all cases investigated, see Figures 20, 21, 22. The amplitudes of $Q_{\mathrm{u}}(t)$, the mass and the SBL are reduced when $Q_{\mathrm{u}}^{\mathrm{r}}(t)$ is set to the constant value corresponding to the mean value of the varying feed point (Figure 21). This may be of importance for the applications, and reducing the variation could be another requirement in a control objective. If, in addition, the regulator gain $K$ is reduced, the amplitude of $Q_{\mathrm{u}}(t)$ is reduced further, but not the mass and the SBL (Figure 22).

For the larger amplitudes of the periodic feed point in Example 2 (Figure 19), the regulator saturates much more, see Figures 24, 25, 26. COSBL1 is satisfied if the regulator gain $K$ is not too small. Although a low value of $K$ means that the SBL touches the bottom periodically in the example in Figure 26, the mean mass and SBL are stabilized, which is difficult to accomplish without a regulator, see the results of the manual control strategies in [4, Figures 9$10]$.

The behaviour of the regulator, when there is a lower bound on the underflow concentration in control objective COSBL2, is illustrated by simulations in Figures 23 and 27. In the former simulation, the control objective is fulfilled despite saturation most of the time. In the latter simulation, the interval of allowable $Q_{\mathrm{u}}(t)$ during the high load intervals is reduced to a single value, because of the hard constraint. This implies that overflow of particles is inevitable. Still, the SBL stays within the thickening zone, in contrast to the case without a regulator, see Figure 19. Another property of these two simulations is that the average mass and SBL are higher than their respective average reference values. The reason is that the mass equals the reference mass at the end of the intervals of low load, and the proportional regulator reduces $Q_{\mathrm{u}}(t)$. To overcome this problem an integrator can be added.

Simulations (not shown here) show that an integrator will also improve the other shown cases in which the average mass is different from its reference value. This occurs as $Q_{\mathrm{u}}^{\mathrm{r}}(t)$ is defined by (5). An advantage then is to avoid or reduce the number of calculations of $Q_{\mathrm{u}}^{\mathrm{r}}(t)$, since this variable is defined implicitly. On the other hand, another parameter, the integrator time, has to be adjusted and preferably anti-windup introduced.

All in all, the presented regulator is not very sensitive to the choices of the reference value $Q_{\mathrm{u}}^{\mathrm{r}}(t)$ and the regulator gain $K$. The behaviour of the process is stabilized significantly in any case.

\section{Acknowledgement}

I am grateful to Dr Ulf Jeppsson, Lund University, for his comments on the manuscript. This work has been supported by the Swedish Research Council (Vetenskapsrådet), project 6212005-3873.

\section{References}

1. S. Diehl. Operating charts for continuous sedimentation I: Control of steady states. J. Eng. Math., 41:117144, 2001.

2. S. Diehl. Operating charts for continuous sedimentation II: Step responses. J. Eng. Math., 53:139-185, 2005.

3. S. Diehl. Operating charts for continuous sedimentation III: Control of step inputs. J. Eng. Math., 54:225259, 2006.

4. S. Diehl. Operating charts for continuous sedimentation IV: Limitations for control of dynamic behaviour. submitted to J. Eng. Math. 
5. S. Diehl. On scalar conservation laws with point source and discontinuous flux function. SIAM J. Math. Anal., 26(6):1425-1451, 1995.

6. S. Diehl. A conservation law with point source and discontinuous flux function modelling continuous sedimentation. SIAM J. Appl. Math., 56(2):388-419, 1996.

7. R. Bürger, K. H. Karlsen, C. Klingenberg, and N. H. Risebro. A front tracking approach to a model of continuous sedimentation in ideal clarifier-thickener units. Nonl. Anal. Real World Appl., 4:457-481, 2003.

8. R. Bürger, K. H. Karlsen, N. H. Risebro, and J. D. Towers. Well-posedness in $B V_{t}$ and convergence of a difference scheme for continuous sedimentation in ideal clarifier-thickener units. Numerische Mathematik, 97:25-65, 2004.

9. K. H. Karlsen and J. D. Towers. Convergence of the Lax-Friedrichs scheme and stability for conservation laws with a discontinuous space-time dependent flux. Chinese Ann. Math. Ser. B., Vol. 25(3):287-318, 2004.

10. G. J. Kynch. A theory of sedimentation. Trans. Faraday Soc., 48:166-176, 1952.

11. R. Bürger, K. H. Karlsen, and J. D. Towers. A model of continuous sedimentation of flocculated suspensions in clarifier-thickener units. SIAM J. Appl. Math., 65:882-940, 2005.

12. R. Bürger, K.H. Karlsen, and J.D. Towers. Mathematical model and numerical simulation of the dynamics of flocculated suspensions in clarifier-thickeners. Chem. Eng. J., 111:119-134, 2005.

13. K. H. Karlsen, N. H. Risebro, and J. D. Towers. Upwind difference approximations for degenerate parabolic convection-diffusion equations with a discontinuous coefficient. IMA J. Numer. Anal., 22:623-664, 2002.

14. K. H. Karlsen, N. H. Risebro, and J. D. Towers. $L^{1}$ stability for entropy solutions of nonlinear degenerate parabolic convection-diffusion equations with discontinuous coefficients. Skr. K. Nor. Vidensk. Selsk., 3:49 pp, 2003.

15. H. Stehfest. An operational dynamic model of the final clarifier. Trans. Inst. Meas. Control, 6(3):160-164, 1984.

16. S. Diehl, G. Sparr, and G. Olsson. Analytical and numerical description of the settling process in the activated sludge operation. In R. Briggs, editor, Instrumentation, Control and Automation of Water and Wastewater Treatment and Transport Systems, pages 471-478. IAWPRC, Pergamon Press, 1990.

17. M. C. Bustos, F. Paiva, and W. Wendland. Control of continuous sedimentation as an initial and boundary value problem. Math. Methods Appl. Sci., 12:533-548, 1990.

18. N. G. Barton, C.-H. Li, and J. Spencer. Control of a surface of discontinuity in continuous thickeners. $J$. Austral. Math. Soc. Ser. B, 33:269-289, 1992.

19. J.-Ph. Chancelier, M. Cohen de Lara, and F. Pacard. Analysis of a conservation PDE with discontinuous flux: A model of settler. SIAM J. Appl. Math., 54(4):954-995, 1994.

20. J.-Ph. Chancelier, M. Cohen de Lara, C. Joannis, and F. Pacard. New insight in dynamic modelling of a secondary settler - II. Dynamical analysis. Wat. Res., 31(8):1857-1866, 1997.

21. S. Diehl. Dynamic and steady-state behaviour of continuous sedimentation. SIAM J. Appl. Math., 57(4):9911018, 1997.

22. T. Gimse and N. H. Risebro. Riemann problems with a discontinuous flux function. In B. Engquist and B. Gustavsson, editors, Third International Conference on Hyperbolic Problems, Theory, Numerical Methods and Applications, volume I, pages 488-502, 1990.

23. S. Diehl. On boundary conditions and solutions for ideal clarifier-thickener units. Chem. Eng. J., 80:119-133, 2000.

24. U. Attir and M. M. Denn. Dynamics and control of the activated sludge wastewater process. AIChE J., 24:693-698, 1978.

25. T. M. Keinath. Operational dynamics and control of secondary clarifiers. J. Water Pollut. Control Fed., 57(7):770-776, 1985.

26. D. Thompson, D. T. Chapmand, and K. L. Murphy. Step feed control to minimize solids loss during storm flows. Res. J. Water Pollut. Control Fed., 61(11-12):1658-1665, 1989.

27. R. R. M. Johnston and K. Simic. Improving thickener operation and control by means of a validated model. Minerals Eng., 4:695-705, 1991.

28. D. Couillard and S. Zhu. Control strategy for the activated sludge process under shock loading. Wat. Res., 26:649-655, 1992.

29. J. C. Kabouris, A. P. Georgakakos, and A. Camara. Optimal control of the activated sludge process: Effect of sludge storage. Wat. Res., 26:507-517, 1992.

30. P. Balslev, C. Nickelsen, and A. Lynggaard-Jensen. On-line flux-theory based control of secondary clarifiers. Wat. Sci. Tech., 30(2):209-218, 1994. 
31. K. Grijspeerdt and W. Verstraete. A sensor for the secondary clarifier based on image analysis. Wat. Sci. Tech., 33(1):61-70, 1996.

32. S.-G. Bergh and G. Olsson. Knowledge based diagnosis of solids-liquid separation problems. Wat. Sci. Tech., 33:219-226, 1996.

33. Y. Kim and W. O. Pipes. Solids routing in an activated sludge process during hydraulic overloads. Water Sci. Tech., 34(3-4):9-16, 1996.

34. U. Nyberg, B. Andersson, and H. Aspegren. Real time control for minimizing effluent concentrations during storm water events. Wat. Sci. Tech., 34:127-134, 1996.

35. Y. L. Sidrak. Control of the thickener operation in alumina production. Control Eng. Practice, 5:1417-1426, 1997.

36. Y. Kim, W. O. Pipes, and P.-G. Chung. Control of activated sludge bulking by operating clarifiers in a series. Wat. Sci. Tech., 38(8-9):1-8, 1998.

37. S. Dauphin, C. Joannis, A. Deguin, G. Bridoux, G. Ruban, and M. Aumond. Influent flow control to increase the pollution load treated during rainy periods. Wat. Sci. Tech., 37(12):131-139, 1998.

38. P. G. Georgieva and S. Feyo De Azevedo. Robust control design of an activated sludge process. Int. J. Robust Nonlinear Control, 9:949-967, 1999.

39. F. Schoenbrunn and T. Toronto. Advanced thickener control. In Advanced Process Control Applications for Industry Workshop, pages 83-86. IEEE Industry Applications Society, 1999.

40. A. Vanderhasselt, B. De Clercq, B. Vanderhaegen, P. Vanrolleghem, and W. Verstraete. On-line control of polymer addition to prevent massive sludge washout. J. Env. Eng., 125:1014-1021, 1999.

41. A. Charef, A. Ghauch, and M. Martin-Bouyer. An adaptive and predictive control strategy for an activated sludge process. Bioprocess Biosystems Eng., 23:529-534, 2000.

42. P. Georgieva and A. Ilchmann. Adaptive lambda-tracking control of activated sludge processes. Int. J. Control, 12:1247-1259, 2001.

43. H. Ito. A dissipative approach to control of biological wastewater treatment plants based on entire nonlinear process models. In Proceedings of the 2004 American Control Conference, volume 6, pages 5489-5495. IEEE, 2004.

44. B.-M. Wilén, D. Lumley, and A. Nordqvist. Dynamics in maximal settling capacity in an activated sludge treatment plant with highly loaded secondary settlers. Wat. Sci. Tech., 50:187-194, 2004.

45. G. Olsson, M. K. Nielsen, Z. Yuan, A. Lynggaard-Jensen, and J.-P. Steyer. Instrumentation, Control and Automation in Wastewater Systems. Intl Water Assn, 2005.

46. U. Jeppsson, C. Rosen, J. Alex, J. Copp, K. V. Gernaey, M.-N. Pons, and P. A. Vanrolleghem. Towards a benchmark simulation model for plant-wide control strategy performance evaluation of WWTPs. Wat. Sci. Tech., 53:287-295, 2006.

47. S. Diehl and U. Jeppsson. A model of the settler coupled to the biological reactor. Wat. Res., 32(2):331-342, 1998.

48. M. V. Maljian and J. A. Howell. Dynamic response of a continuous thickener to overloading and underloading. Trans. Ind. Chem. Eng., 56:55-61, 1978. 